Aus der Klinik für Thorax-, Herz- und Gefäßchirurgie
(Prof. Dr. Dipl.-Phys. F. A. Schöndube)
im Zentrum Chirurgie
der Medizinischen Fakultät der Universität Göttingen

\title{
Korrektur der Fallotschen Tetralogie nach vorhergehender Palliativoperation -Langzeitergebnisse aus einem historischen Patientenkollektiv
}

\author{
INAUGURAL- DISSERTATION \\ zur Erlangung des Doktorgrades \\ der Medizinischen Fakultät der \\ Georg- August- Universität zu Göttingen
}

vorgelegt von

Kathrin Annelore Nowak, geb. Rottwinkel

aus

Göttingen

Göttingen 2015 
Dekan:

Prof. Dr. rer. nat. H. K. Kroemer

I. Berichterstatter/in: Prof. Dr. med. Theodoros Tirilomis

II. Berichterstatter/in: Prof. Dr. Matthias Sigler

III. Berichterstatter/in: Prof. Dr. Samuel Tobias Sossalla

IV. Promotorvertretung: Prof. Dr. Thomas Meyer

Tag der mündlichen Prüfung: 19.05.2016 


\section{Inhaltsverzeichnis}

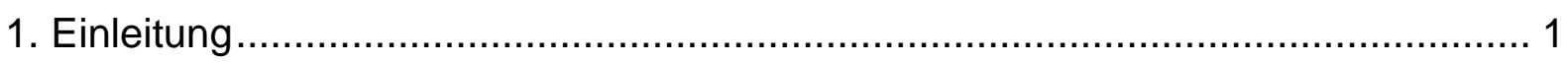

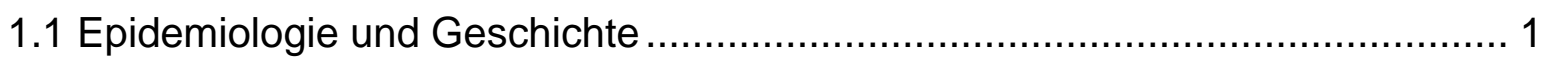

1.2 Hämodynamik und Pathophysiologie ..................................................... 2

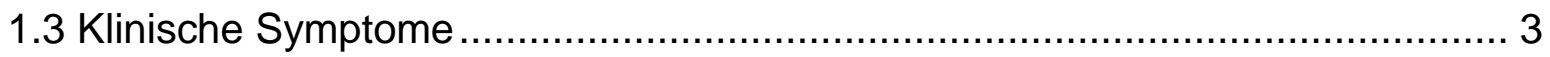

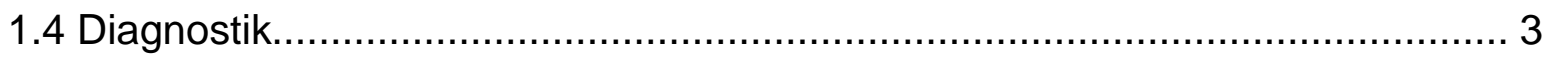

1.5 Behandlung der Fallotschen Tetralogie ................................................. 5

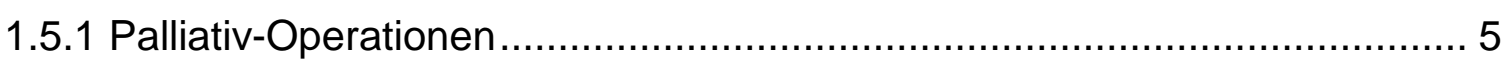

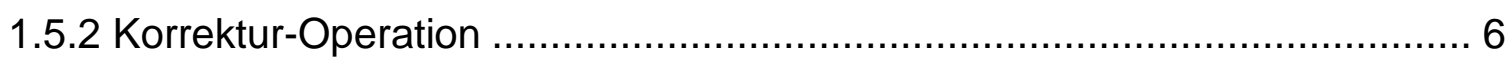

1.6 Ziel der vorliegenden Arbeit...................................................................... 7

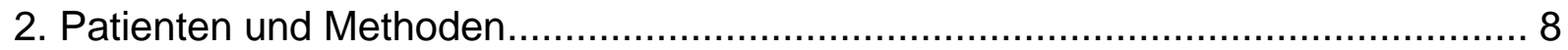

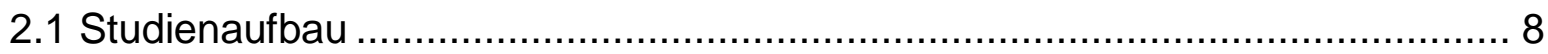

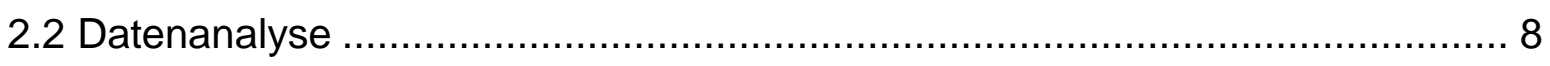

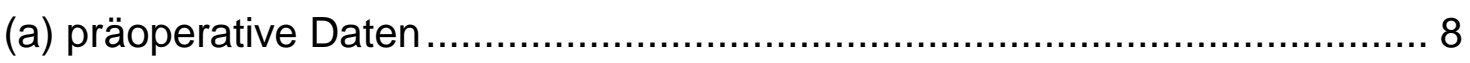

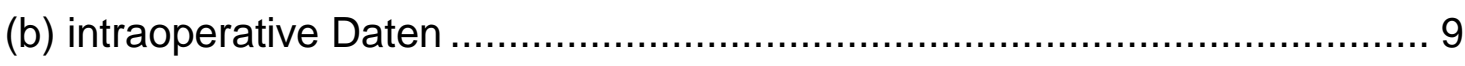

(c) postoperative Daten ……............................................................. 9

(d) Daten im prä- und postoperativen Vergleich ......................................... 9

(e) Langzeitdaten (Follow up): ................................................................ 9

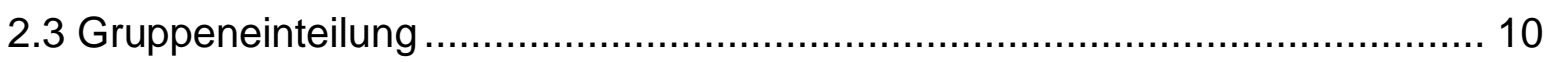

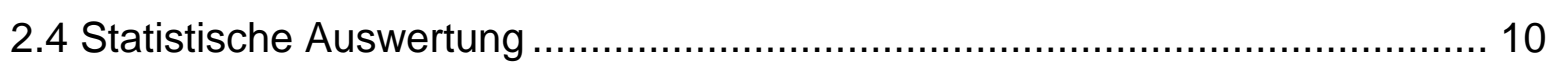

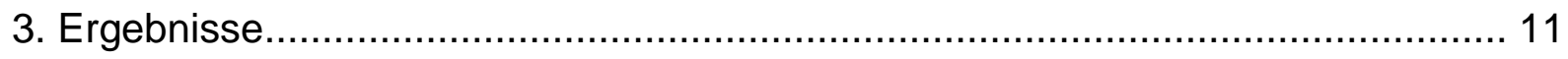

3.1 Epidemiologische Daten und Palliativ-Operationen.................................... 11

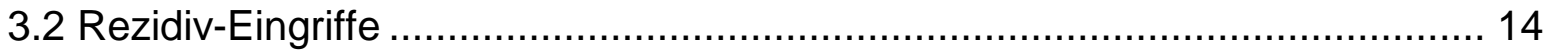

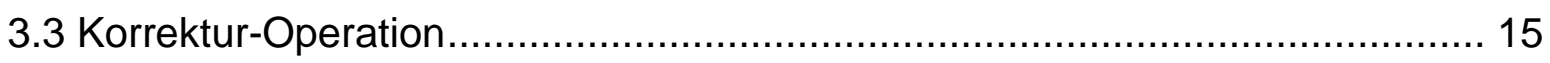

3.3.1 Alter zur Korrektur-Operation ....................................................... 15

3.3.2 Intervall zwischen Palliativ-Operation und Korrektur .............................. 17

3.3.3 Intraoperative Daten bei der Korrektur-Operation .................................... 19

3.4. Frühpostoperative Daten nach der Korrektur-Operation ............................... 20

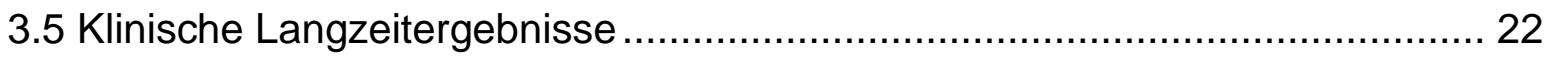

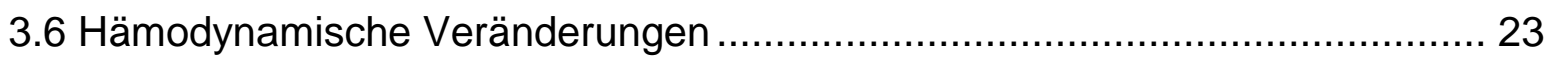

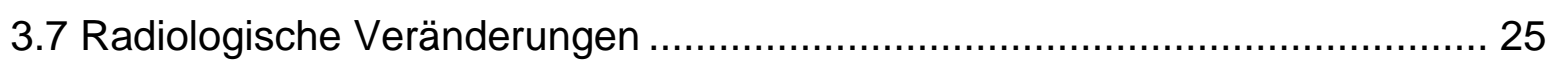

3.8 Veränderungen des Lagetyps im EKG .................................................. 25

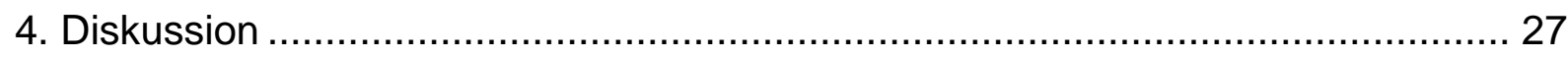

4.1 Indikationen zur operativen Behandlung ……......................................... 27 


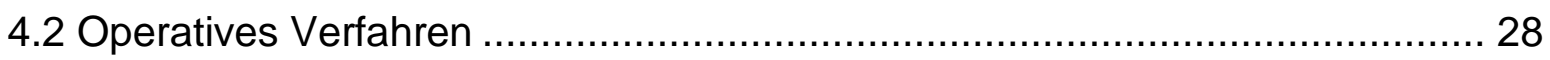

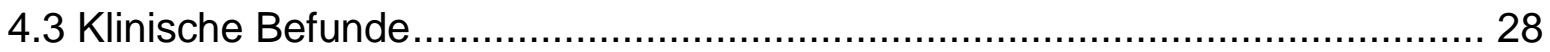

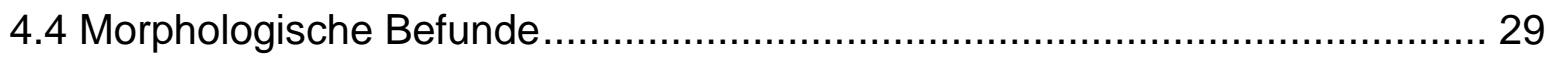

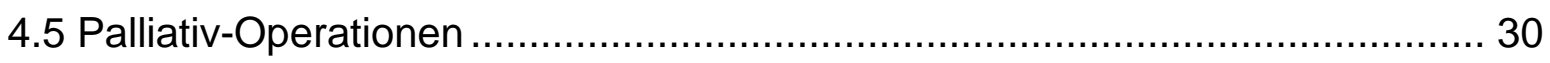

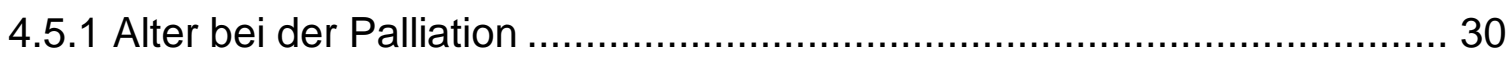

4.5.2 Zentraler vs. peripherer Shunt........................................................ 30

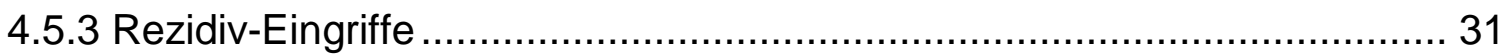

4.6 Korrektur-Operation nach vorheriger Palliation ......................................... 32

4.6.1 Intervall zwischen Palliativ- und Korrektur-Operation............................... 32

4.6.2 Operationstechniken der anschließenden Korrektur-Operation ................ 32

4.6.3 Operationsdauer bei der Korrektur-Operation ......................................... 33

4.7 Frühletalität und -morbidität nach „gestufter“ Korrektur ................................ 34

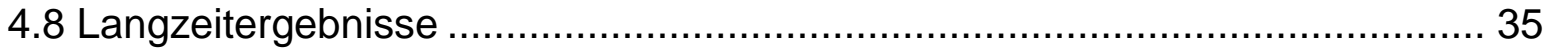

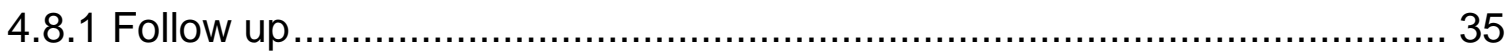

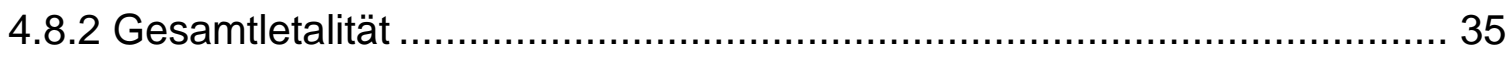

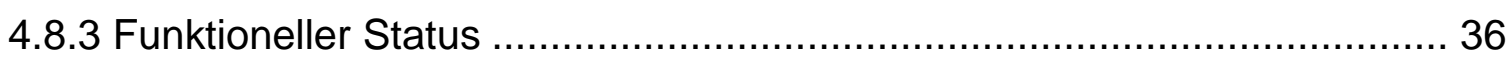

4.9 Verlaufsanalyse und Risikofaktoren ....................................................... 36

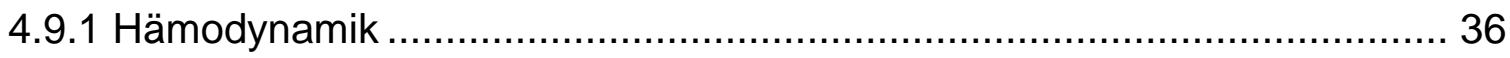

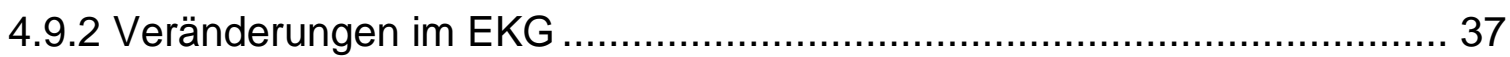

4.9.3 Bedeutung des Herz-Thorax-Quotienten (HTQ)................................ 37

4.10 Bedeutung der vorliegenden Arbeit und Aussicht auf die Zukunft ................ 38

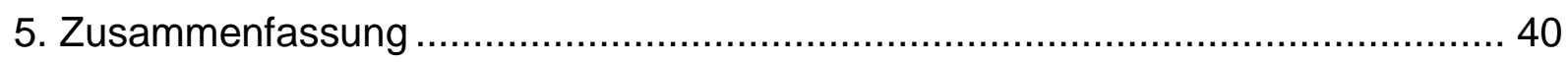

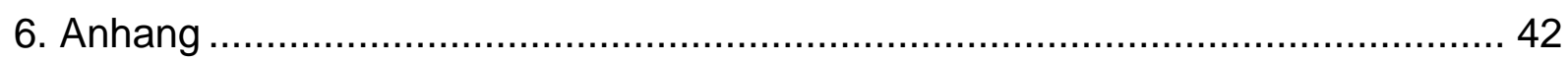

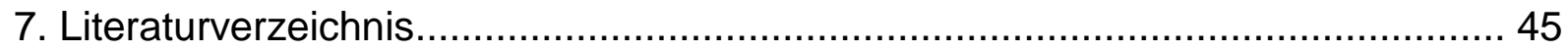




\section{Abbildungsverzeichnis}

Abb.1.1 Morphologie eines Herzens mit Fallotscher Tetralogie............. 1

Abb.1.2 Röntgen-Thorax eines Herzens mit Fallotscher Tetralogie........... 4

Abb.1.3 Korrektur der Fallotschen Tetralogie mit VentrikelseptumdefektPatchverschluß mit (a) subvalvulärem Patch, (b) transvalvulärem Patch und (c) klappentragenden extrakardialen Conduit.......... 7

Abb. 3.1 Einteilung der Patienten nach der ersten Palliativ-Operation........ 11

Abb. 3.2 Verteilung der Palliativ-Operationen vor der Korrektur-Operation in den verschiedenen Gruppen

Abb. 3.3 Altersverlauf zum Zeitpunkt der Korrektur-Operation in 5-JahresSchritten.

Abb. 3.4 Altersdurchschnitt der beiden Gruppen zum Zeitpunkt der Korrektur-Operation.

Abb. 3.5 Alter bei der Korrektur-OP in den verschiedenen Gruppen, in 5-Jahres-Schritten

Abb. 3.6 Intervall zwischen Palliativ-OP und Korrektur-OP beim Gesamtkollektiv, unterteilt in 5-Jahres-Schritte.

Abb. 3.7 Intervall zwischen Palliativ-OP und Korrektur-OP

Abb. 3.8 Häufigkeiten bezüglich der Durchführung von Kommissurotomie, Infundibulektomie und Erweiterungsplastik mit Patch im Rahmen der Korrektur-Operation

Abb. 3.9 NYHA-Stadium der Gruppen beim letzten Kontakt.

Abb. 3.10 Veränderung des rechtsventrikulären/ linksventrikulären Druckquotienten im Vergleich zwischen prä- und postoperativ in der BTA- und WCA-Gruppe

Abb. 3.11 Der Lagetyp vor der Korrekturoperation in den verschiedenen Gruppen nach der Palliativ-Operation.

Abb. 3.12 Der Lagetyp nach der Korrektur in den verschiedenen Gruppen (gemäß der vorherigen Palliativ-Operation) 


\section{$\underline{\text { Tabellenverzeichnis }}$}

Tabelle 2.1 Lagetypeinteilung durch Berechnung des alpha-Winkels...... 9

Tabelle 3.1 Klinische Symptome der Patienten vor der Palliativ-Operation. 12

Tabelle 3.2 Präoperative Hämoglobin- und Hämatokritwerte.............. 12

Tabelle 3.3 Verteilung der assoziierten Vitien präoperativ.................. 13

Tabelle 3.4 Art der Pulmonalstenose vor der ersten Palliativ- Operation.... 13

Tabelle 3.5 Intensivaufenthalt nach der Korrektur-Operation (in Stunden

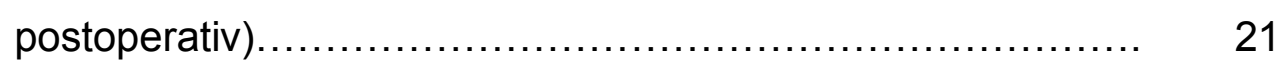

Tabelle 3.6 Veränderung der Ventrikeldrücke (in $\mathrm{mmHg}$ ) im Prä-und PostOP- Vergleich............................................ 24 


\section{Abkürzungsverzeichnis}

A.

Abb.

AD

Ao

ASD

BTA

dl

EKG

$\mathrm{g}$

h

HTQ

ICU

LA

LV

LVP

$\mathrm{ml}$

$\mathrm{mmHg}$

$\mathrm{n}$

NYHA

$P A$

PDA

RA

RV

RVP

Syst.

V.

WCA
Arterie

Abbildung

andere

Aorta

Atrium-Septum-Defekt

Blalock-Taussig-Anastomose

Deziliter

Elektrokardiogramm

Gramm

Stunde

Herz-Thorax-Quotient

Intensiv care unit - Intensivstation

linkes Atrium

linker Ventrikel

linksventrikulärer Druck

Milliliter

Millimeter Quecksilbersäule

Anzahl

New York Heart Association

Pulmonalarterie

persistierender Ductus arteriosus

rechtes Atrium

rechter Ventrikel

rechtsventrikulärer Druck

systolisch

Vene

Waterston-Cooley-Anastomose 


\section{Einleitung}

\subsection{Epidemiologie und Geschichte}

Die Fallotsche Tetralogie zählt mit 8-10\% aller angeborenen Herzfehler insgesamt zu den fünf häufigsten kardialen angeborenen Missbildungen (Bailliard und Anderson 2009). Unter den zyanotischen Herzfehlern ist die Fallotsche Tetralogie die häufigste zyanotische Herzfehlbildung (Gelb et al. 1991).

Auf diese Herzmissbildung wurde bereits im Jahre 1777 von dem holländischen Arzt Edmund Sandifort hingewiesen. Zu dieser Zeit bestätigt eine Vielzahl von Veröffentlichungen seine Beobachtungen wie zum Beispiel die von William Hunter 1784. Aber erst der französische Arzt Étienne Louis Arthur Fallot fügte die vier Hauptmerkmale in seiner Beschreibung der "Cyanose cardiaque“ im Jahre $1888 \mathrm{zu}$ einem Syndrom zusammen und gab inm den Namen blue malady. Maude Abbot prägte 1924 den Begriff "tetralogy of Fallot“ (van Praagh 2009).

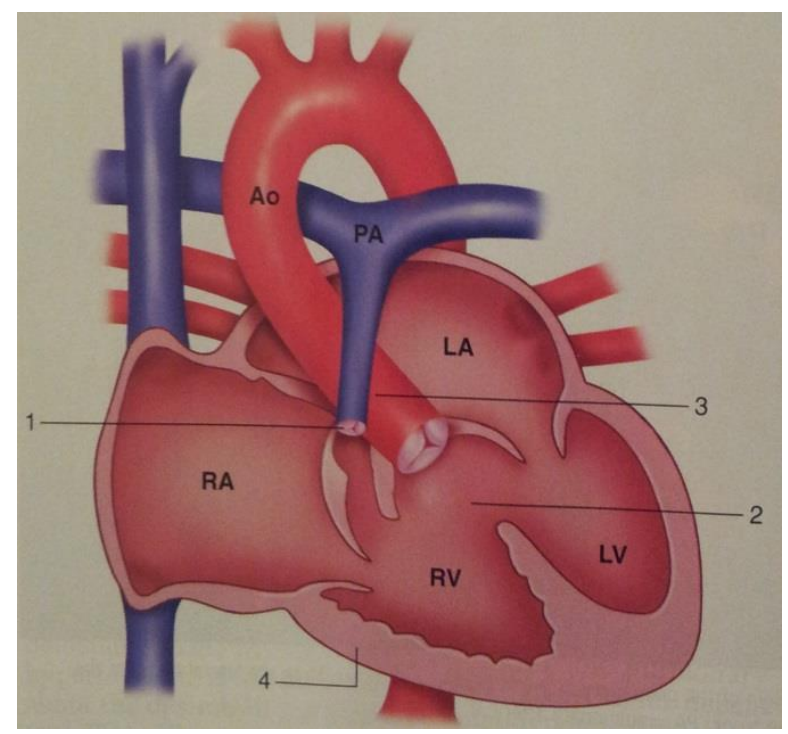

Abb.1.1 Morphologie eines Herzens mit Fallotscher Tetralogie; 1 Pulmonalstenose; 2 Ventrikelseptumdefekt; 3 überreitende Aorta; 4 rechtsventrikuläre Hypertrophie. $A o=$ Aorta; $L A=$ linkes Atrium; $L V=$ linker Ventrikel; $P A=$ Pulmonalarterie; $R A=$ rechtes Atrium; $\mathrm{RV}=$ rechter Ventrikel (mit freundlicher Genehmigung des Wiley- Liss. Verlags: Webb et al. 2008, S. 1588) 
Der Herzfehlerkomplex besteht aus vier morphologischen Korrelaten (s. Abb. 1.1):

1) einer Pulmonalstenose

2) einem Ventrikelseptumdefekt

3) einer ante- und dextroponierten Aortenwurzel und

4) einer Hypertrophie des rechten Ventrikels

Die Ursachen für die Fehlbildung bei der Fallotschen Tetralogie werden als multifaktoriell angesehen. Für eine mögliche genetische Komponente sprechen das gehäufte familiäre Auftreten und eine Korrelation mit anderen Chromosomenanomalien wie zum Beispiel der Trisomie 21 (Marino et al. 1996). Aber auch Umweltfaktoren, maternale Phenylketonurie (Siwik et al 2001) und Erkrankung mit dem Rötelvirus während der Schwangerschaft gelten als begünstigende Faktoren für diese Fehlbildung (Siwik et al. 2001, Bailliard und Anderson 2009).

\subsection{Hämodynamik und Pathophysiologie}

Die Hämodynamik und die Pathophysiologie sind besonders von zwei Missbildungen beeinflusst, dem Ventrikelseptumdefekt und der Pulmonalstenose. Durch den Ventrikelseptumdefekt besteht auf Ventrikelebene ein Shunt. Aufgrund der Größe des Defektes und dem dadurch annähernden Druckausgleich in beiden Ventrikeln wird die Shuntrichtung durch den herrschenden Ausflusswiderstand in dem jeweiligen Ventrikel bestimmt. Damit ist im Falle der Fallotschen Tetralogie bei einer schweren Pulmonalstenose auch der Ausflusswiderstand des rechten Ventrikels deutlich erhöht, und es resultiert ein besonders großer rechts-links-gerichteter Shunt. Für die Widerstandserhöhung ist es dabei irrelevant, wo sich die Pulmonalstenose befindet, ob infundibulär, valvulär, supravalvulär oder kombiniert. Der Grad der überreitenden Aorta hat ebenfalls keinen Einfluss auf die Shuntrichtung oder das Shuntvolumen. Damit ist der Schweregrad der Pulmonalstenose der Hauptindikator für die Klinik. Mit dem Grad des Rechts-Links-Shunts steigt die Minderversorgung des arteriellen Blutes mit Sauerstoff, und es erhöht sich der Grad der Zyanose. Ist der Ausflusswiderstand rechts nur gering ausgebildet, kann es auch zu einem 
bidirektionalen, selten zu einem links-rechts-gerichteten Shunt kommen. In diesem Fall handelt es sich um einen sogenannten „Pink Fallot“. (Duro et al. 2010).

Der rechte Ventrikel ist aufgrund der Pulmonalstenose stärker druckbelastet als es bei einem Normalherz der Fall ist. Der Muskel reagiert mit einer Hypertrophie. Mit der Hypertrophie wird das Erregungsleitungssystem des Herzens verändert. Der Lagetyp ändert sich zu Gunsten des Rechtstypen und es kommt zu einem Rechtsschenkelblock. Es zeigen sich im Verlauf der Erkrankung vermehrt Arrhythmien, die bis zum plötzlichen Herztod (Sudden Cardiac Death) führen können (Nollert et al. 2003).

\subsection{Klinische Symptome}

Während initial klinisch vor allem eine Belastungsdyspnoe auftritt, leiden die Kinder im Verlauf an Entwicklungsstörungen, einer Trinkschwäche und schließlich an einer schweren Zyanose. Eine besonders schwere Komplikation sind die hypoxischen Anfälle. Dabei kommt es zu einer akuten Verminderung der Lungendurchblutung und dadurch zu einem zunehmenden Rechts-Links-Shunt, wohingegen der arterielle Blutdruck während des Anfalls gleich bleibt. Der hypoxische Zustand mit gelegentlicher kurzzeitiger Bewusstlosigkeit bis hin zum Tod wird dabei vermutlich durch eine vermehrte Kontraktion der rechten Ausflusstraktmuskulatur ausgelöst (Duro et al. 2010, Rudolph 2001). Durch die „Hockstellung“ können die hypoxischen Anfälle kupiert werden. Der Mechanismus dabei ist durch eine entstehende Erhöhung des Widerstandes im Körperkreislauf zu erklären. Dieser vermindert den Rechts-Links-Shunt und verbessert damit die Lungenperfusion (Duro et al. 2010).

Als Folge der Zyanose entwickelt sich eine kompensatorische Polyglobulie, welche zu einer erhöhten Viskosität des Blutes und damit zu einer erhöhten Thrombosegefahr führt. Eine über einen längeren Zeitraum bestehende Zyanose führt zu der Entstehung der typischen Trommelschlegelfinger, Uhrglasnägel und einer Gingivahyperplasie.

\subsection{Diagnostik}

Auskultatorisch findet sich, vor allem bei ausgeprägten infundibulären Pulmonalstenosen, ein spindelförmiges Systolikum über dem 3. und 4. Interkostalraum links parasternal sowie ein gespaltener zweiter Herzton mit einem betonten Aorten- 
klappenschlusston bei einem stark abgeschwächt hörbaren bzw. fehlenden Pulmonalklappenschlusston im 2. Interkostalraum.

Im Röntgen-Thorax zeigt sich die typische Herzkonfiguration der Fallotschen Tetralogie meist noch nicht bei den Säuglingen. Erst im Kindesalter bildet sich die entsprechende Herzsilhouette aus. Dabei handelt es sich um das so genannte Holzschuhherz ("Coeur de sabot") mit einer angehobenen Herzspitze und einer ausgeprägten Herztaille. (Abb. 1.2) Die Silhouette ist vom Ausprägungsgrad der Obstruktion des rechtsventrikulären Ausflusstraktes abhängig. Bei ca. einem Viertel der Patienten bildet sich der Aortenbogen rechts ab und es zeigt sich eine vermehrte Transparenz der Lungenperipherie als Zeichen der pulmonalen Minderdurchblutung (Bailliard und Anderson 2009).

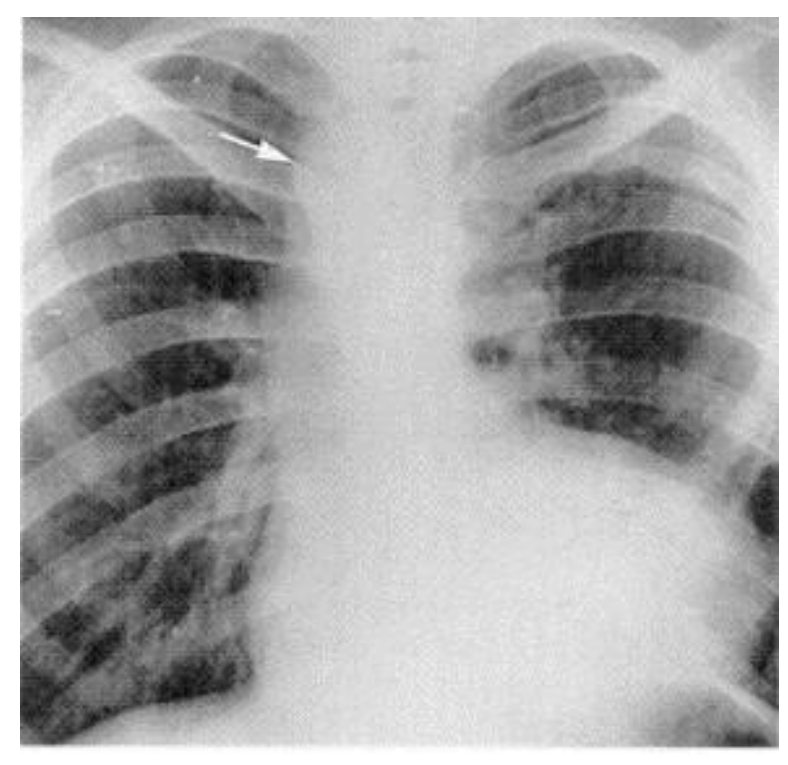

Abb.1.2 Röntgen-Thorax eines Herzens mit Fallotscher Tetralogie. Pfeil: rechtsseitig positionierter Aortenbogen (right aortic arch) (mit freundlicher Genehmigung des Elsevier Verlags: Baron 1999, S. 412)

Die Echokardiographie ist in den meisten Fällen ausreichend, um die Diagnose der Fallotschen Tetralogie zu stellen, da man den Ventrikelseptumdefekt, den Status der Pulmonalklappe und den obstruktiven rechtsventrikulären Ausflusstrakt meist problemlos darstellen kann. Es wird empfohlen, vor allem präoperativ, eine Herzkatheteruntersuchung durchzuführen, um gegebenenfalls aorto-pulmonale Kollaterale und assoziierte Koronararterienanomalien darzustellen. Als charakteristischen manometrischen Herzkatheterbefund bei der Fallotschen Tetralogie findet 
man, bedingt durch den Ventrikelseptumdefekt, einen systolischen Druckausgleich in beiden Ventrikeln. Der systolische Druckgradient zwischen rechtem Ventrikel und der Pulmonalarterie korrespondiert mit dem Grad der rechtsventrikulären Ausflusstraktobstruktion (Bailliard und Anderson 2009).

\subsection{Behandlung der Fallotschen Tetralogie}

Ohne Korrektur-Operation sterben $70 \%$ der Patienten bis zum zehnten Lebensjahr (Babu-Narayan und Gatzoulis 2011). Die außerordentlich ungünstige Prognose der Fallotschen Tetralogie verdeutlicht die Notwendigkeit der chirurgischen Therapie.

Die Korrektur-Operation setzt jedoch (aufgrund der intrakardialen Intervention) die mögliche Eröffnung des Herzens voraus. Wenn das aus technischen Gründen nicht realisierbar ist oder der erforderliche Einsatz der Herz-Lungen-Maschine nicht möglich ist, stehen einige Palliativeingriffe zur Verfügung.

\subsubsection{Palliativ-Operationen}

\section{a) Shuntanlage}

Die amerikanische Kinderärztin H. B. Taussig entdeckte, dass die klinischen Symptome bei den Kindern mit einem zyanotischen Herzvitium und einem zusätzlich offenen Ductus Botalli deutlich geringer ausgeprägt waren als bei den gleichen Herzvitien mit geschlossenem Ductus Botalli. Ihre Idee war, eine Shunt-Operation durchzuführen und damit die hämodynamischen Verhältnisse eines offenen Ductus Botalli zu imitieren. Mit dieser Idee überwies sie den ersten Patienten an den Chirurgen Alfred Blalock. (Taussig 1947)

(I) Blalock-Taussig-Operation:

Blalock führte am 29.11.1944 die erste Anastomosenoperation zwischen der linken A. subclavia und der linken A. pulmonalis durch. Hierbei stellte er eine End-zu-SeitVerbindung zwischen der A. subclavia und der Pulmonalarterie her, die auch rechtsseitg durchgeführt werden kann. (Blalock und Taussig 1984) 
(II) Waterston-Cooley-Anastomose:

Waterston entwickelte 1962 eine weitere Modifikation der Shuntoperation. Er stellte in einem extrakardialen Eingriff eine Verbindung der Aorta ascendens mit der rechten Pulmonalarterie her. (Waterston 1962)

Diese Methode wurde von Cooley und Hallmann 1966 weiter modifiziert. Im Gegensatz zu dem bei Waterston rechtsseitigen posterolateralen Zugang, mit Erreichen der Aorta und A. pulmonalis hinter der V. cava superior, wählte Cooley ebenfalls eine rechtsseitige, jedoch streng anterolaterale Thorakotomie und konnte die Präparation der Gefäße vor der V. cava superior durchführen. (Cooley und Hallman 1966)

(III) Potts-Smith-Anastomose:

Potts und Smith modifizierten die Blalock-Taussig-Anastomose, indem sie eine Anastomose zwischen der thorakalen Aorta und dem extraperikardialen Anteil der linken Pulmonalarterie anfertigten (Potts et al. 1946).

\section{b) Dilatation: Erweiterung der rechten Ausflussbahn nach Sellors/Brock}

Sellors und Brock entwickelten die Operationsmethode unabhängig voneinander. Hier war das Ziel, die Pulmonalstenose direkt anzugehen. Dabei erfolgte eine geschlossene Erweiterung des rechtsventrikulären Ausflusstraktes: Durch die Vorderwand bzw. die rechtsventrikuläre Spitze wurde ein Valvutom zur Pulmonalstenose vorgeschoben und anschließend eine geschlossene Sprengung durchgeführt. (Sellors 1948, Brock 1950)

\subsubsection{Korrektur-Operation}

Die erste Korrektur-Operation wurde erst nach Entwicklung und Einsatz der Extrakorporalen Zirkulation 1954 von dem amerikanischen Herzchirurgen R. C. Lillehei und seinen Mitarbeitern durchgeführt (Lillehei et al. 1955). Das Verfahren der Korrektur-Operation blieb bis heute mit einigen Modifikationen unverändert:

Der große Ventrikelseptumdefekt wird durch einen Patch verschlossen, die Ausflussbahnrekonstruktion erfolgt durch Infundibuluminzision bzw.- resektion und Erweiterungsplastik, je nach zugrundeliegender Anatomie (Abb 1.3). Nach Bedarf erfolgt eine Valvulotomie oder Kommissurotomie. 


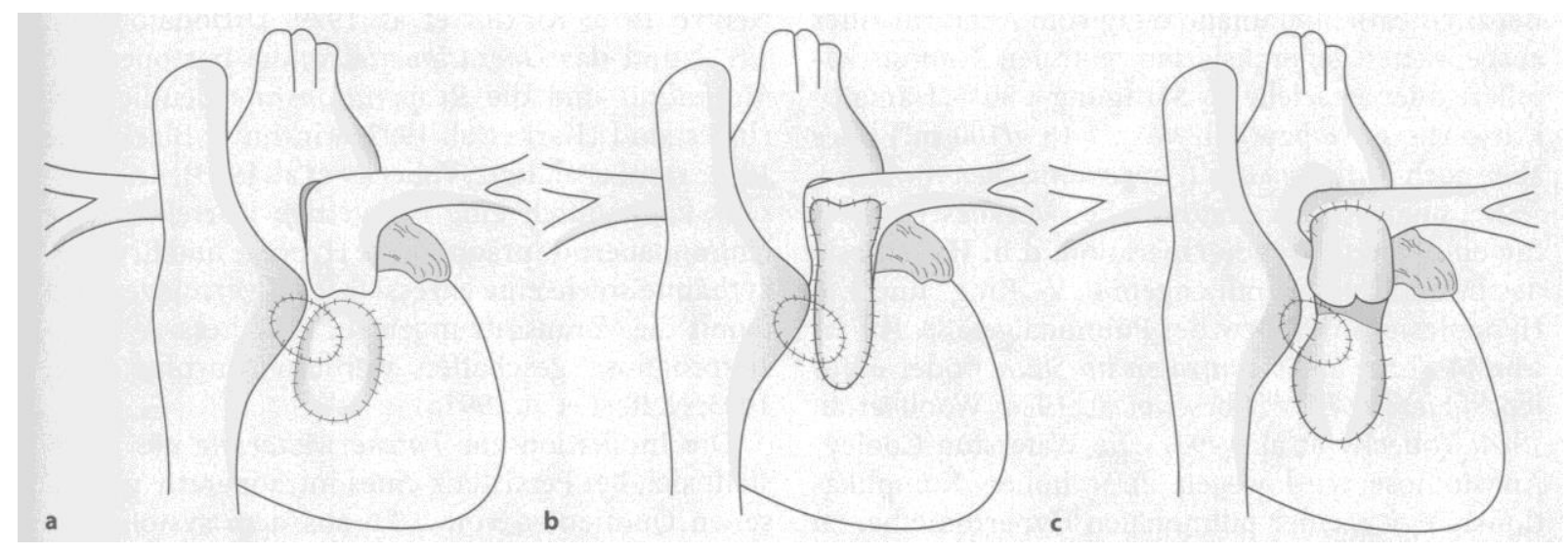

Abb.1.3 Korrektur der Fallotschen Tetralogie mit Ventrikelseptumdefekt-Patchverschluß mit (a) subvalvulärem Patch, (b) transvalvulärem Patch und (c) klappentragendem extrakardialem Conduit (mit freundlicher Genehmigung des Springer Verlags aus: Schumacher et al 2001, S. 296)

\subsection{Ziel der vorliegenden Arbeit}

Seit 1960 werden Patienten mit einer Fallotschen Tetralogie in der Klinik für ThoraxHerz- und Gefäßchirurgie der Universität Göttingen operiert. Der Einsatz der HerzLungen-Maschine war in der frühen Phase der Behandlung älteren Patienten vorbehalten. Erst durch zunehmende Miniaturisierung der zur Verfügung stehenden Systeme konnten auch zunehmend jüngere Patienten einer Korrektur-Operation unterzogen werden. Dadurch erhielten in den ersten Jahrzehnten die meisten Patienten zuerst eine Palliativ-Operation und erst danach die Korrektur-Operation.

Ziel der vorliegenden Arbeit ist es, die Langzeitergebnisse dieser Patienten, die in den ersten 24 Jahren (1960-1984) zuerst eine palliative Operation erhalten haben und erst im Verlauf die Korrektur des Herzfehlers erhalten haben, zu erfassen und zu analysieren.

Hierzu wurden die unterschiedlichen Palliativ-Operationstechniken berücksichtigt und bewertet. 


\section{Patienten und Methoden}

\subsection{Studienaufbau}

Bei der vorliegenden Arbeit handelt es sich um die retrospektive Untersuchung eines historischen Patientenkollektivs.

Im Zeitraum von 1960-1984 wurden in der Thorax-, Herz- und Gefäßchirurgie in Göttingen 691 Patienten mit der Diagnose Fallotsche Tetralogie operiert. Patienten mit einer primären Korrektur (311 Patienten) wurden von der Studie ausgeschlossen. Bei weiteren 56 Patienten waren die Akten nicht auffindbar und keine ausreichenden Daten vorhanden, deswegen wurden diese Patienten ebenfalls aus der Studie ausgeschlossen. Somit konnten insgesamt 324 Patienten in die Studie eingeschlossen werden.

\subsection{Datenanalyse}

Die Datenerhebung erfolgte retrospektiv anhand eines eigens hierfür zusammengestellten Erfassungsbogens, der in drei große Abschnitte (präoperative Daten, intraoperative Daten und postoperative Daten) unterteilt wurde. Folgende Daten wurden analysiert:

(a) präoperative Daten

- Alter

- Geschlecht

- Körperoberfläche

- Lagetyp im EKG (Tabelle 2.1)

- Klinische Daten: Dyspnoe, Zyanose, hypoxämische Anfälle

- Laborchemische Werte (Hämoglobin, Hämatokrit)

- Herzmorphologie (insbes. Art der Pulmonalstenose)

- assoziierte Vitien

- Hämodynamische Parameter (LV-Druck, RV-Druck) 
Tabelle 2.1 Lagetypeinteilung durch Berechnung des alpha-Winkels

\begin{tabular}{|l|l|}
\hline Lagetyp & Winkel alpha \\
\hline Indifferenztyp in \% & $30^{\circ}$ bis $60^{\circ}$ \\
\hline Steiltyp in \% & $60^{\circ}$ bis $90^{\circ}$ \\
\hline Rechtstyp in \% & $90^{\circ}$ bis $120^{\circ}$ \\
\hline Linkstyp in \% & $30^{\circ}$ bis $-30^{\circ}$ \\
\hline überdrehter Linkstyp in \% & $<-30^{\circ}$ \\
\hline überdrehter Rechtstyp in \% & $>120^{\circ}$ \\
\hline Sagittaltyp in \% & $\begin{array}{l}\text { Drehung um die Transversal- } \\
\text { achse (alpha meistens nicht } \\
\text { bestimmbar) }\end{array}$ \\
\hline
\end{tabular}

\section{(b) intraoperative Daten}

- Konfiguration der Pulmonalgefäße bei Korrektur-Operation

- Art der Durchführung der Korrektur-Operation

- Operationsdauer der Korrektur-Operation

(c) postoperative Daten

- Intensivaufenthalt

- Blutverluste

- Intubationsdauer

- Re-Thorakotomie

- Frühletalität(Sterberate innerhalb der ersten 30 Tage nach der Korrektur-Operation)

(d) Daten im prä- und postoperativen Vergleich

- Verlauf der Hämodynamik

- Änderungen des Druckquotienten

- Verlauf des Herzthoraxquotienten

- Lagetypänderung

(e) Langzeitdaten (Follow up):

- Funktioneller Status beim letzten Kontakt (NYHA-Klasse)

- Letalität

- Hämodynamische Parameter (LV-Druck, RV-Druck) 


\subsection{Gruppeneinteilung}

Anhand der ersten Palliativ-Operation wurden 3 Gruppen gebildet: 1) die BlalockTaussig-Gruppe (BTA-Gruppe), 2) die Waterston-Cooley-Anastomosen-Gruppe (WCA-Gruppe) und 3) eine zusammengefasste Gruppe (AD-Gruppe="Andere" Gruppe) aus unterschiedlichen anderen Palliativ-Operationen: der PottsAnastomose, einem Pulmonalisbanding nach Müller-Damm, einer Brockschen Operation und dem Einsetzen eines Metallbügels. Da es sich bei der dritten Gruppe um eine Mischung aus verschiedenen Operationsmethoden mit jeweils einer geringen Anzahl an Patienten handelte, wurden diese Patienten zwar im Gesamtkollektiv der Vollständigkeit halber berücksichtigt, aber nicht in der weiteren Subgruppenanalyse.

\subsection{Statistische Auswertung}

Die statistische Analyse erfolgte unter Zuhilfenahme des Computerprogramms Statistika (Statsoft, Version 5). Die Daten der Patienten wurden in die einzelnen Gruppen eingeteilt und die Mittelwerte (MW) und deren mittlere Standardfehler (SEM) berechnet. Mittels des Student'schen T-Tests wurden die statistischen Signifikanzen zwischen den prä- und postoperativen Parametern in den einzelnen Gruppen ermittelt. Um die Unterschiede zwischen den Gruppen zu überprüfen, wurde die ANOVA (Analysis of Variance) angewandt. Signifikant waren dabei die Differenzen mit einer Irrtumswahrscheinlichkeit von $p<0,05$. 


\section{Ergebnisse}

\subsection{Epidemiologische Daten und Palliativ-Operationen}

Von den 324 Patienten mit Fallotscher Tetralogie, die in die Auswertung mit einbezogen wurden, waren 180 Patienten männlichen (56\%) und 144 Patienten weiblichen Geschlechts (44\%).

Der Altersdurchschnitt der Patienten zum Zeitpunkt der durchgeführten KorrekturOperation betrug insgesamt $8,7 \pm 4,7$ Jahre.

Die 324 Patienten konnten anhand der ersten durchgeführten Palliativ-Operation in drei Gruppen unterteilt werden. In der ersten Gruppe befinden sich alle Patienten, die eine Blalock-Taussig-Anastomose (BTA-Gruppe) als Palliativ-Operation erhalten haben. Diese Gruppe umfasst 250 Patienten (77,2\%) und ist damit die größte Gruppe. Die Patienten der zweiten Gruppe erhielten als erste Palliativ-Operation eine Waterston-Cooley-Anastomose (WCA-Gruppe). Insgesamt handelt es sich hierbei um 57 Patienten (17,6\%). In den 1960ern wurde die Waterston-Cooley-Anastomose nicht durchgeführt, erst ab 1970 fand sie Anwendung in unserem Patientenkollektiv.

Die restlichen 17 Patienten (5,2\%) erhielten viele verschiedene Palliativeingriffe und wurden als „Andere“-Gruppe (AD-Gruppe) zusammengefasst (Abb. 3.1). Da diese Gruppe klein und sehr inhomogen ist, werden diese Daten lediglich im Anhang dargestellt (s. Anhang 1- 3).

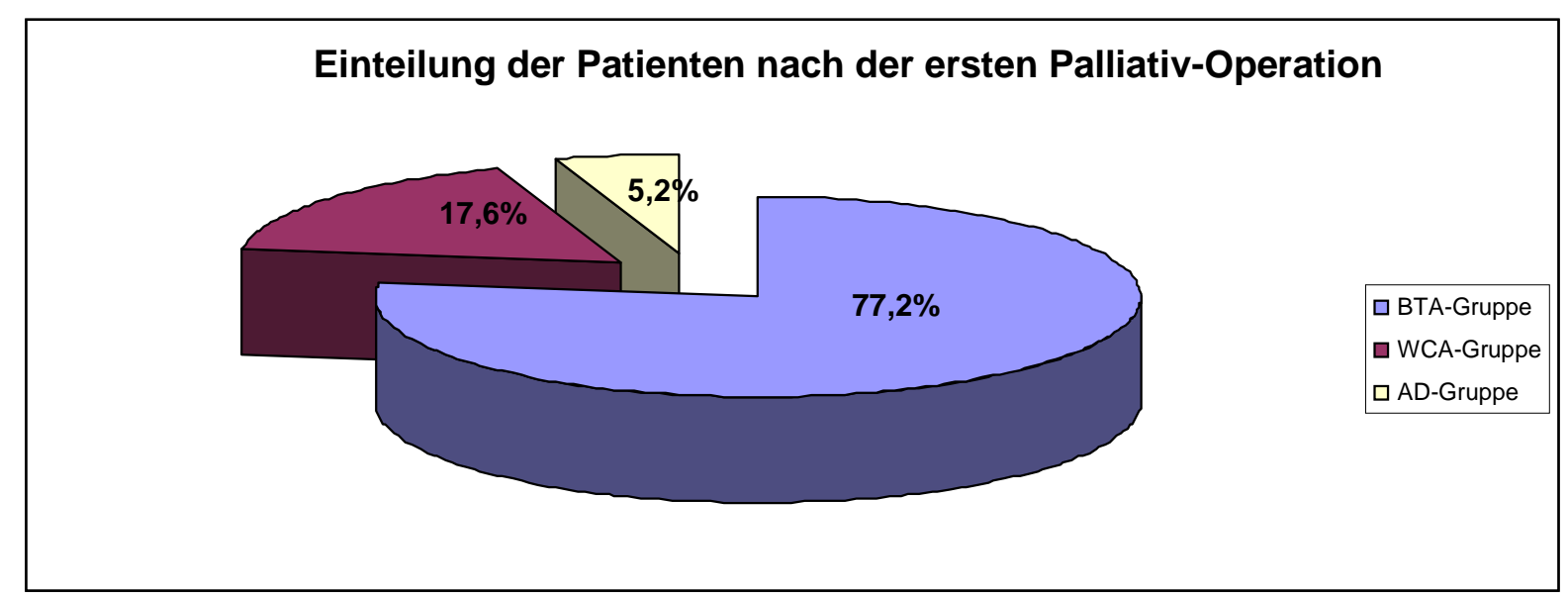

Abb. 3.1 Einteilung der Patienten nach der ersten Palliativ-Operation (in Prozent) 
In Tabelle 3.1 sind die klinischen präoperativen Parameter der verschiedenen Gruppen dargestellt. Das führende Symptom war die Zyanose mit einer Häufigkeit von über $90 \%$. Hypoxische Anfälle hatten insgesamt $12 \%$ der untersuchten Patienten.

Tabelle 3.1 Klinische Symptome der Patienten vor der Palliativ-Operation

\begin{tabular}{|l|l|l|l|l|l|l|}
\hline Klinik & \multicolumn{2}{|l|}{$\begin{array}{l}\text { alle Patienten } \\
(\mathrm{n}=324)\end{array}$} & \multicolumn{2}{l|}{$\begin{array}{l}\text { BTA } \\
(\mathrm{n}=250)\end{array}$} & \multicolumn{3}{l|}{$\begin{array}{l}\text { WCA } \\
(\mathrm{n}=57)\end{array}$} \\
\hline & $\mathbf{n}$ & $\%$ & $\mathbf{n}$ & $\%$ & $\mathrm{n}$ & $\%$ \\
\hline Dyspnoe & 121 & 37,3 & 101 & 40,4 & 14 & 24,6 \\
\hline Zyanose & 292 & 90,1 & 230 & 92 & 51 & 89,5 \\
\hline $\begin{array}{l}\text { hypoxische } \\
\text { Anfälle }\end{array}$ & 39 & 12 & 27 & 10,8 & 11 & 19 \\
\hline
\end{tabular}

Der präoperative Hämoglobinwert lag bei 17,3g/dl, der Hämatokritwert entsprechend durchschnittlich bei 50,7\%. Der Hämatokrit und der Hämoglobinwert waren bei den BTA-Patienten höher im Vergleich zu der WCA- Gruppe (Tabelle 3.2).

Tabelle 3.2 Präoperative Hämoglobin- und Hämatokritwerte

\begin{tabular}{|l|l|l|l|}
\hline & $\begin{array}{l}\text { alle OP } \\
(n=324)\end{array}$ & $\begin{array}{l}\text { BTA } \\
(n=250)\end{array}$ & $\begin{array}{l}\text { WCA } \\
(n=57)\end{array}$ \\
\hline Hb (g/dl) & $17,3( \pm 3,0)$ & $17,6( \pm 3,0)$ & $16,1( \pm 2,6)$ \\
\hline Hkt (\%) & $50,7( \pm 8,9)$ & $51,6( \pm 8,8)$ & $48( \pm 8,2)$ \\
\hline
\end{tabular}

Ein persistierender Ductus arteriosus Botalli (PDA) wurde insgesamt bei $5 \%$ der Patienten gefunden, ein Atrium-Septum-Defekt (ASD) trat bei 11\% der Patienten auf (Tabelle 3.3). 
Tabelle 3.3 Verteilung der assoziierten Vitien präoperativ

\begin{tabular}{|c|c|c|c|c|c|c|c|c|}
\hline $\begin{array}{l}\text { assoziierte } \\
\text { Vitien }\end{array}$ & \multicolumn{2}{|c|}{$\begin{array}{l}\text { alle Patienten } \\
(n=324)\end{array}$} & \multicolumn{2}{|c|}{$\begin{array}{l}\text { BTA } \\
(n=250)\end{array}$} & \multicolumn{2}{|c|}{$\begin{array}{l}\text { WCA } \\
(n=57)\end{array}$} & \multicolumn{2}{|c|}{$\begin{array}{l}\text { andere } \\
(n=17)\end{array}$} \\
\hline & $\mathbf{n}$ & $\%$ & $\mathbf{n}$ & $\%$ & $\mathbf{n}$ & $\%$ & $\mathbf{n}$ & $\%$ \\
\hline $\begin{array}{l}\text { PDA } \\
\end{array}$ & 15 & 5 & 8 & 3 & 6 & 11 & 1 & 6 \\
\hline ASD & 36 & 11 & 26 & 10,4 & 2 & 3,5 & 0 & 0 \\
\hline
\end{tabular}

Die valvuläre Pulmonalstenose war die häufigste Form der Stenose bei den Patienten mit einer Shunt-Operation. Die Verteilung der Pulmonalstenosen ist in der Tabelle 3.4 detailliert aufgeführt.

Tabelle 3.4 Art der Pulmonalstenose vor der ersten Palliativ-Operation

\begin{tabular}{|l|l|l|l|l|l|l|}
\hline $\begin{array}{l}\text { Pulmonal- } \\
\text { stenose }\end{array}$ & \multicolumn{2}{|l|}{$\begin{array}{l}\text { alle OP } \\
(\mathbf{n = 3 2 4 )}\end{array}$} & \multicolumn{2}{l|}{$\begin{array}{l}\text { BTA } \\
(\mathbf{n}=\mathbf{2 5 0})\end{array}$} & \multicolumn{2}{l|}{$\begin{array}{l}\text { WCA } \\
(\mathbf{n}=57)\end{array}$} \\
\hline & $\mathbf{n}$ & $\%$ & $\mathbf{n}$ & $\%$ & $\mathbf{n}$ & $\%$ \\
\hline valvulär & 134 & 41,4 & 98 & 39,2 & 34 & 59,7 \\
\hline subvalvulär & 48 & 14,8 & 38 & 15,2 & 5 & 8,8 \\
\hline $\begin{array}{l}\text { valvulär und } \\
\text { subvalvulär }\end{array}$ & 28 & 8,6 & 26 & 10,4 & 2 & 3,5 \\
\hline $\begin{array}{l}\text { 3-fach (sub-, } \\
\text { supra- und } \\
\text { valvulär) }\end{array}$ & 3 & 0,9 & 2 & 0,8 & 0 & 0 \\
\hline Atresie & 15 & 4,6 & 9 & 3,6 & 6 & 10,5 \\
\hline keine & 10 & 3,1 & 8 & 3,2 & 1 & 1,8 \\
\hline $\begin{array}{l}\text { keine } \\
\text { Angaben }\end{array}$ & 86 & 26,5 & 69 & 27,6 & 9 & 15,8 \\
\hline
\end{tabular}




\subsection{Rezidiv-Eingriffe}

Vor der Korrektur-Operation wurde bei $17 \%$ aller untersuchten Patienten eine zweite Palliativ-Operation und bei $1 \%$ eine dritte Palliativ-Operation durchgeführt. Nach einer BTA erfolgten eine kontralaterale BTA bei $11 \%$ der Patienten, sowie eine WCA bei $3 \%$. Nach einer WCA als primäre Palliativ-Operation, wurde bei $21 \%$ eine anschließende BTA durchgeführt. Und bei einem Patienten noch eine dritte PalliativOperation mit einer BTA auf der kontralateralen Seite (Abbildung 3.2).

Im Durchschnitt betrug das mittlere Zeitintervall bis zur 2. Palliativ-Operation 38,5 $( \pm 32,2)$ Monate. In der BTA Gruppe lag der Durchschnitt knapp darüber mit 39,1 $( \pm 33,3)$ Monaten und in der WCA Gruppe minimal niedriger mit 37 Monaten $( \pm 30,6)$ im Durchschnitt.

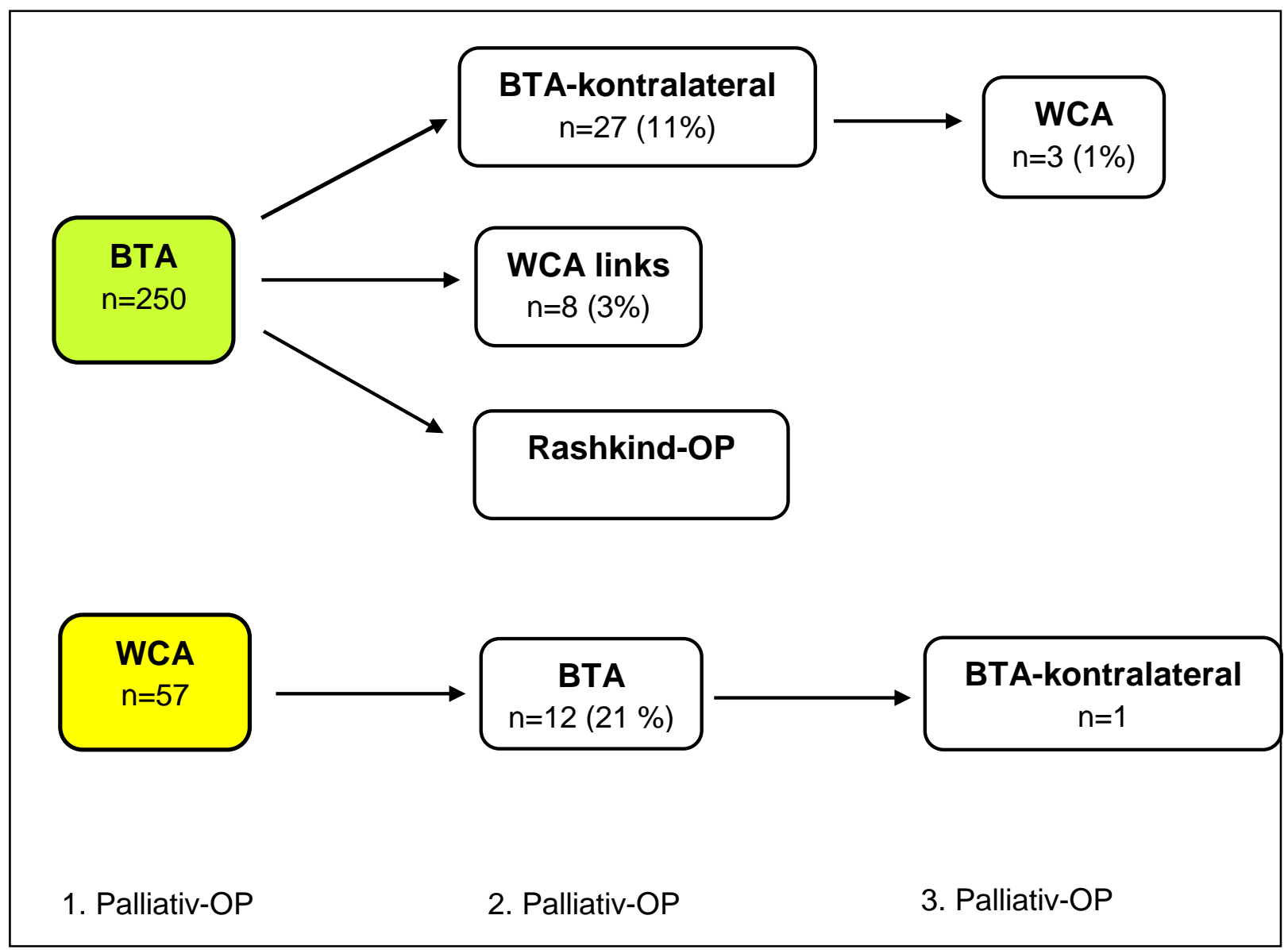

Abb.3.2 Verteilung der Palliativ-Operationen vor der Korrektur-Operation in den verschiedenen Gruppen 


\subsection{Korrektur-Operation}

\subsubsection{Alter zur Korrektur-Operation}

Von 1960-1964 liegt das mittlere Alter zum Zeitpunkt der Korrektur-Operation aller Patienten, die zuvor eine Palliativ-OP bekommen haben, bei 132 Monaten $(=11$ Jahre). Ende der 60er Jahre steigt das Alter zur Korrektur-Operation leicht an auf durchschnittlich 141 Monate (=11 2/3 Jahre) und bis zum Ende der 70er Jahre kommt es zu einem Absinken des Durchschnittsalters auf 84 Monate ( $=7$ Jahre; Abb. 3.3). In den Jahren 1980-84 ist das Durchschnittsalter bei der Korrektur-Operation wieder leicht angestiegen auf durchschnittlich 93 Monate (=7,75 Jahre).

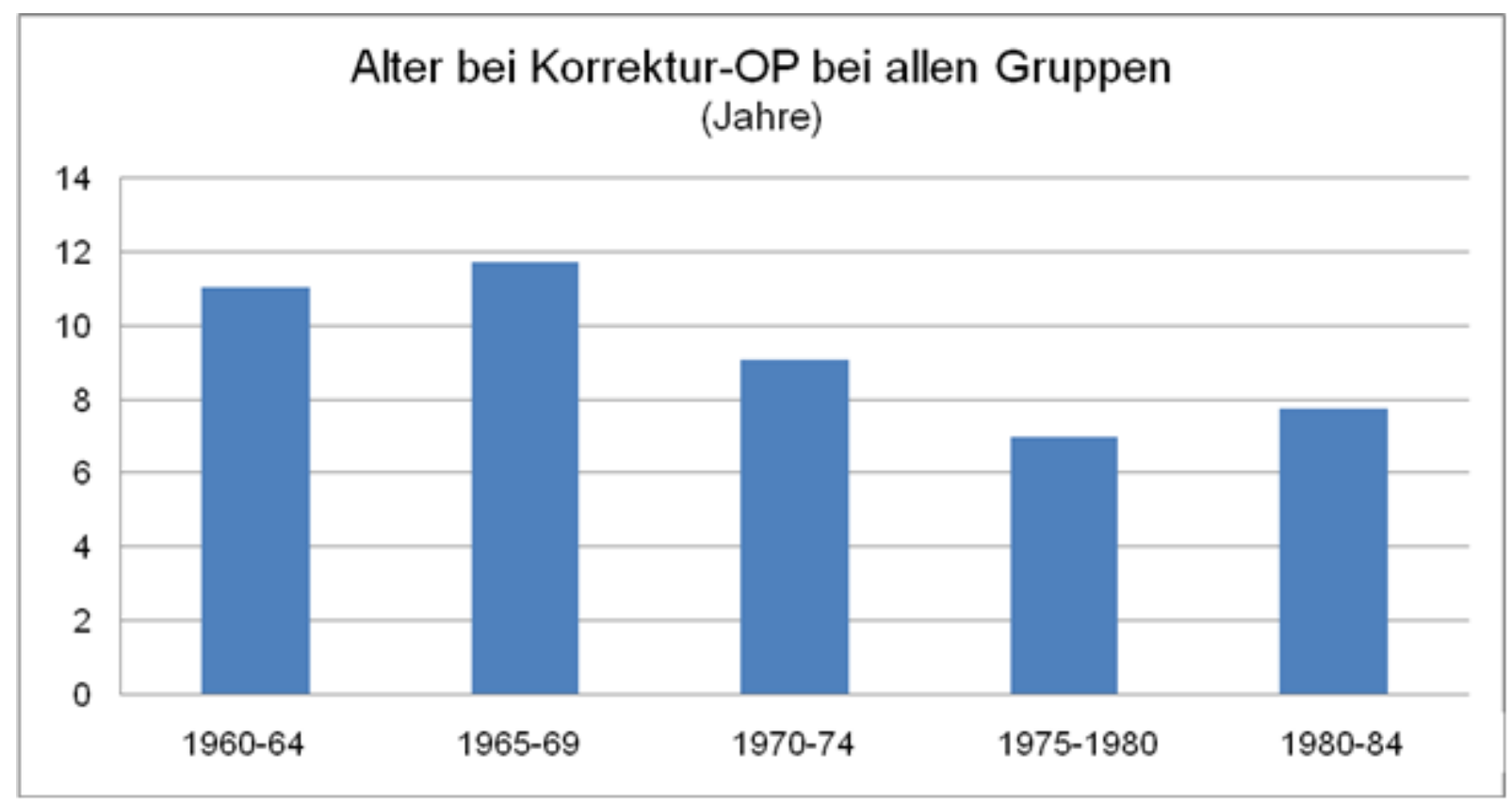

Abb.3.3 Altersverlauf zum Zeitpunkt der Korrektur-Operation in 5-Jahres-Schritten

Insgesamt lag in der BTA-Gruppe das mittlere Alter bei 8,45 $( \pm 4,62)$ Jahren, die Kinder in der WCA-Gruppe waren im Durchschnitt zum Operationszeitpunkt mit 6,89 $( \pm 2,96)$ Jahren deutlich jünger ( $p=0,015 ;$ Abb. 3.4). 


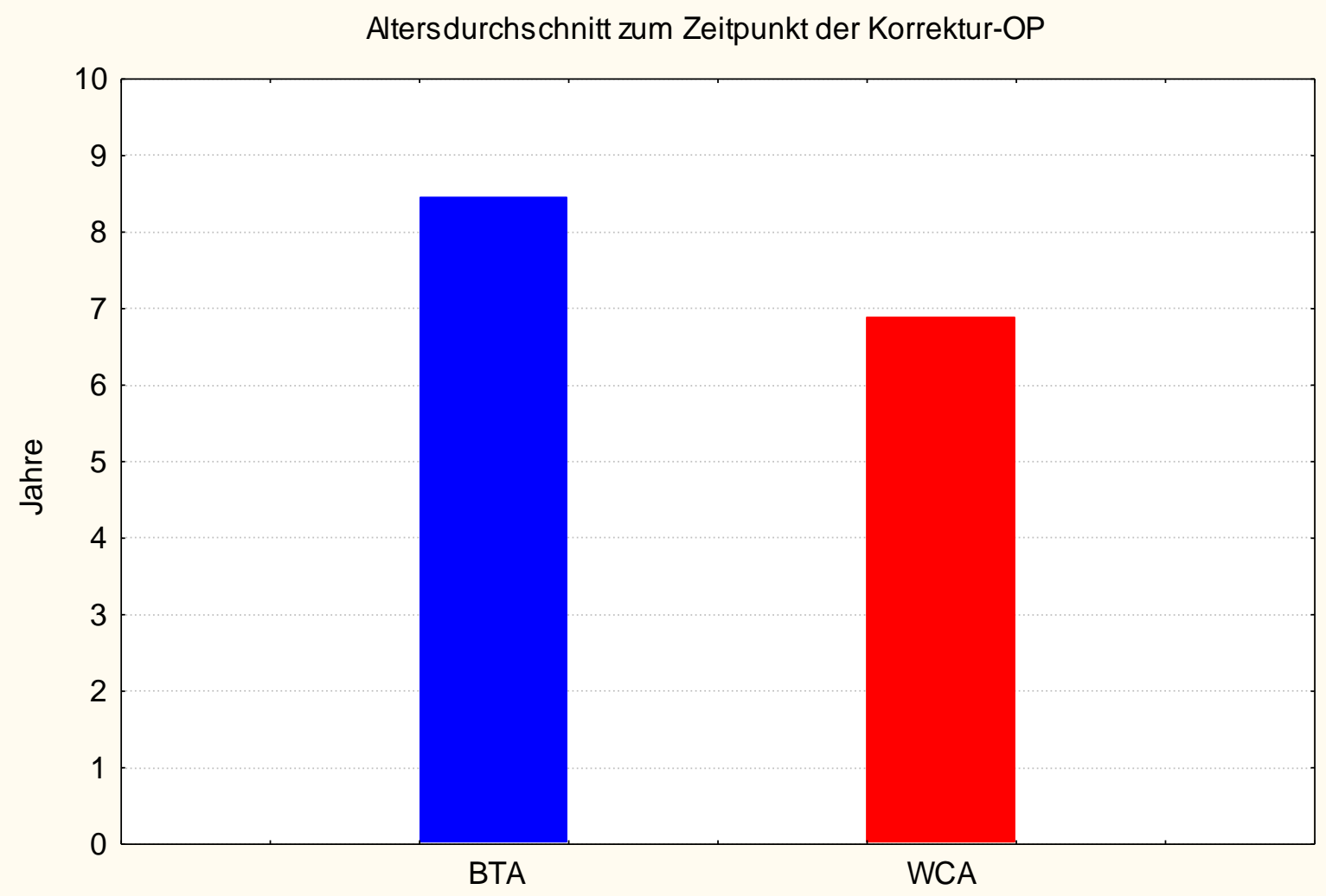

Abb. 3.4 Altersdurchschnitt der beiden Gruppen zum Zeitpunkt der Korrektur-Operation

Die Entwicklung innerhalb der Gruppen verhält sich vergleichbar zum Gesamtkollektiv, wobei hier zu berücksichtigen ist, dass die WCA in den 1960er Jahren nicht angelegt wurde (Abb. 3.5). Ende der 70er Jahre kommt es zu einem Absinken des mittleren Alters (in der BTA- Gruppe auf 6,5 Jahren). In den 1980ern steigt das Durchschnittsalter bei der Korrektur-Operation nach vorheriger BTA wieder an, während es in der WCA-Gruppe zu einer weiteren Reduktion des mittleren Alters auf 5,7 Jahre kommt. 
Alter bei Korrektur-OP in den Gruppen

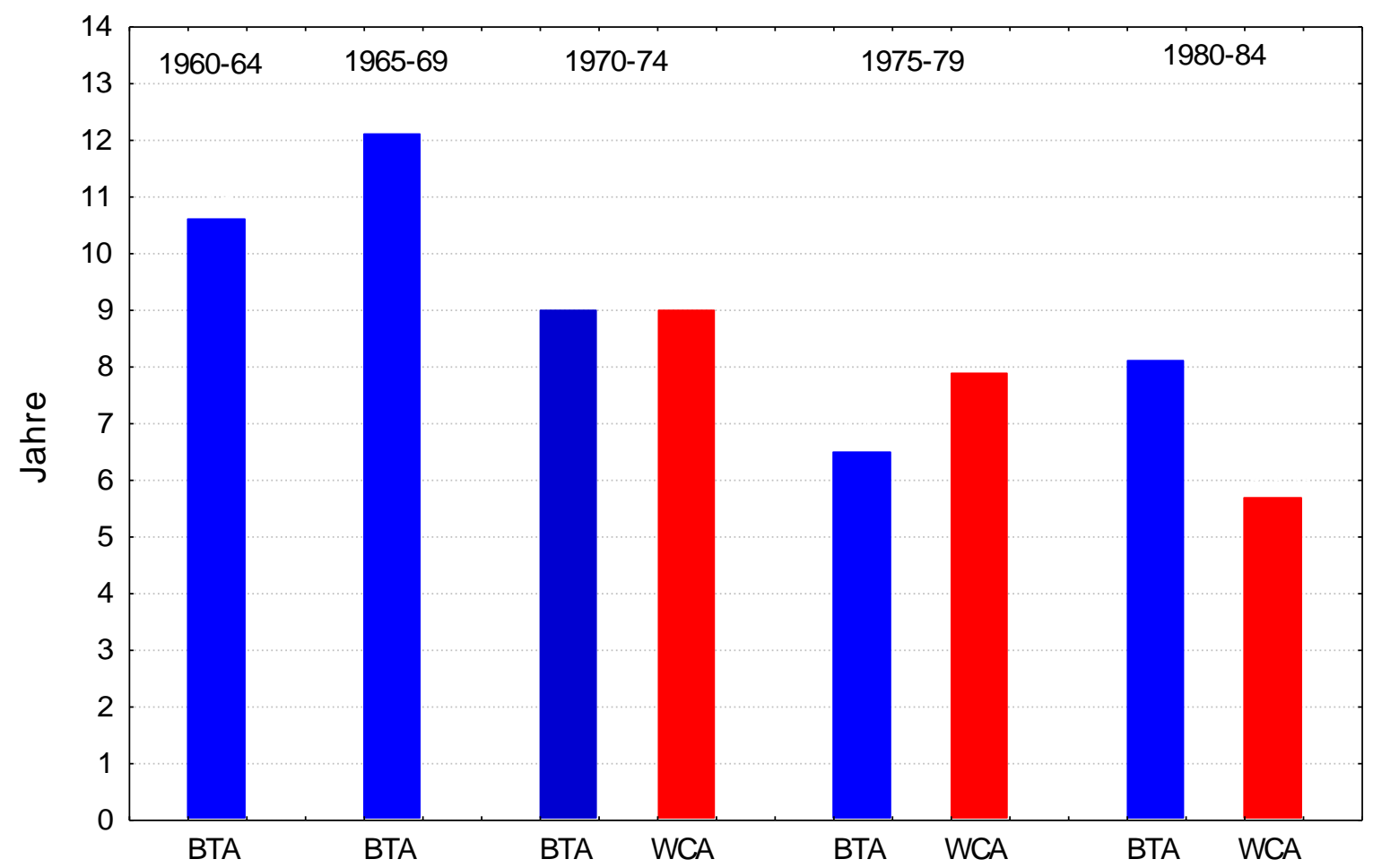

Abb. 3.5 Alter bei der Korrektur-OP in den verschiedenen Gruppen, in 5-Jahres-Schritten

\subsubsection{Intervall zwischen Palliativ-Operation und Korrektur}

Anfang der 60er Jahre zeigt sich ein durchschnittliches Zeitintervall zwischen der 1. Palliativ- und der abschließenden Korrektur-Operation von 67,7 \pm 33 Monate, das entspricht ca. 5 1/2 Jahren. Das Zeitintervall steigt Ende der 60er Jahre an bis auf durchschnittlich 86,4 \pm 46 Monate. In den 60er Jahren wurde jedoch, wie bereits erwähnt, nur die BTA durchgeführt. Die WCA wurde erst in den 1970ern durchgeführt. Anfang der 70er Jahre ist das Intervall zwischen Palliation und Korrektur deutlich gesunken mit einem Durchschnittswert von $55 \pm 27$ Monaten, das entspricht $4 \quad 1 / 2$ Jahren. Zum Ende der 70er Jahre steigt das Intervall auf durchschnittlich 62 Monate. 


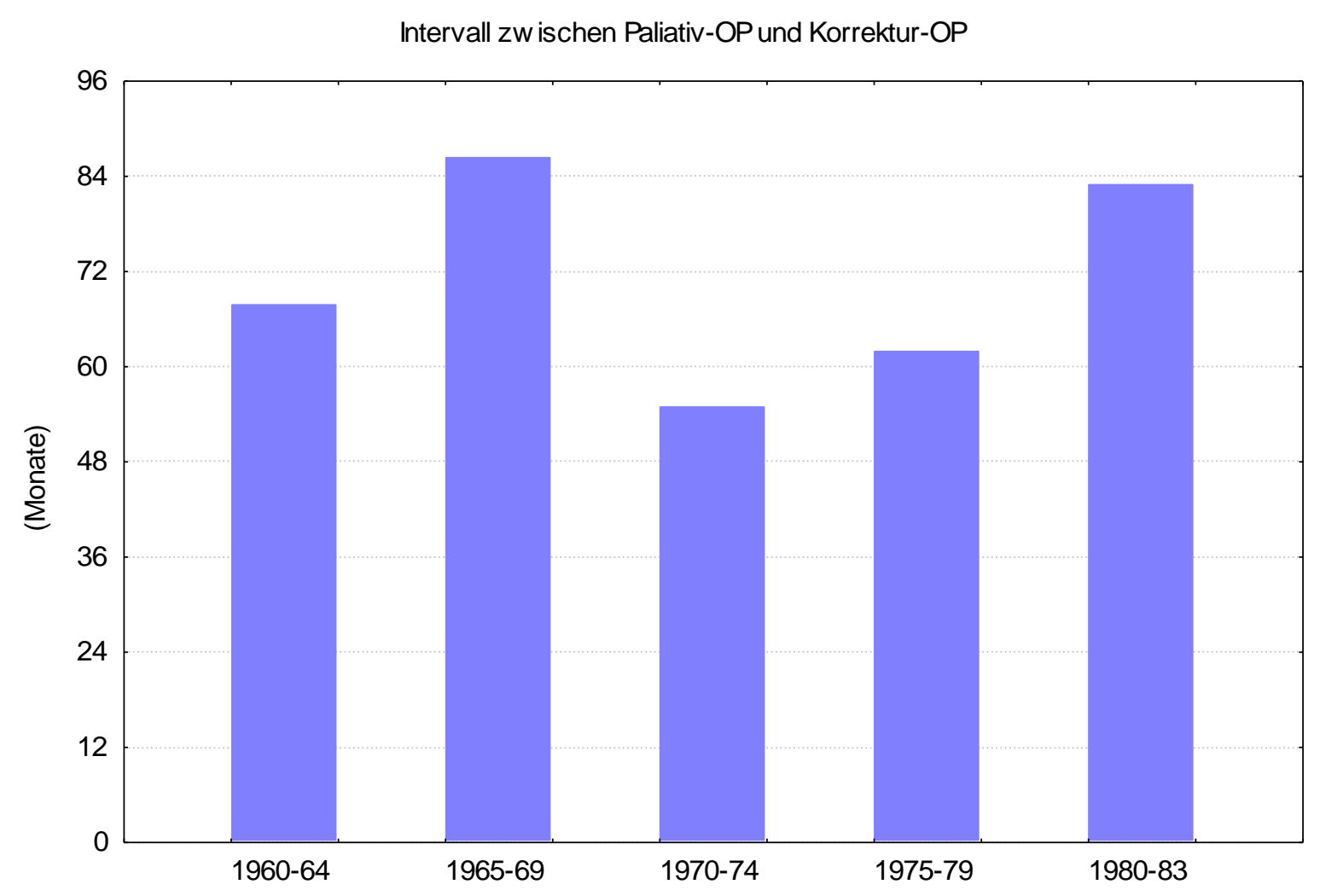

Abb. 3.6 Intervall zwischen Palliativ-OP und Korrektur-OP beim Gesamtkollektiv, unterteilt in 5-JahresSchritte

Innerhalb der Gruppen zeigen sich teilweise deutliche Unterschiede (Abb. 3.7): In der BTA-Gruppe mit einem durchschnittlichen Intervall von 53 Monaten (= 4,4 Jahre) und in der WCA-Gruppe mit durchschnittlich 76 Monaten $(=6,3$ Jahre; $p=0,1)$. Anfang der 70er Jahre ist das Intervall zwischen Palliation und Korrektur in der BTA-Gruppe 55 Monate und in der WCA-Gruppe 57 Monate. Ein signifikanter Unterschied der Intervalle zeigte sich von 1975-1979 mit p= 0,042 (BTA-Gruppe: 52,5 Monate, WCAGruppe: 75,5 Monate). Von 1980-1984 kam es wieder zu einem deutlichen Anstieg des Zeitintervalls mit einem Durchschnittswert für alle Operationen von 83 Monaten (=6,9 Jahre). Auch hier gab es einen tendentiellen Gruppenunterschied, die BTAGruppe hatte insgesamt ein Intervall von 78 Monaten $(=6,5$ Jahre) und die WCAGruppe von 93 Monaten ( $=7,8$ Jahre; $p=0,38)$. 
Intervall zwischen Palliativ-OP und Korrektur-OP

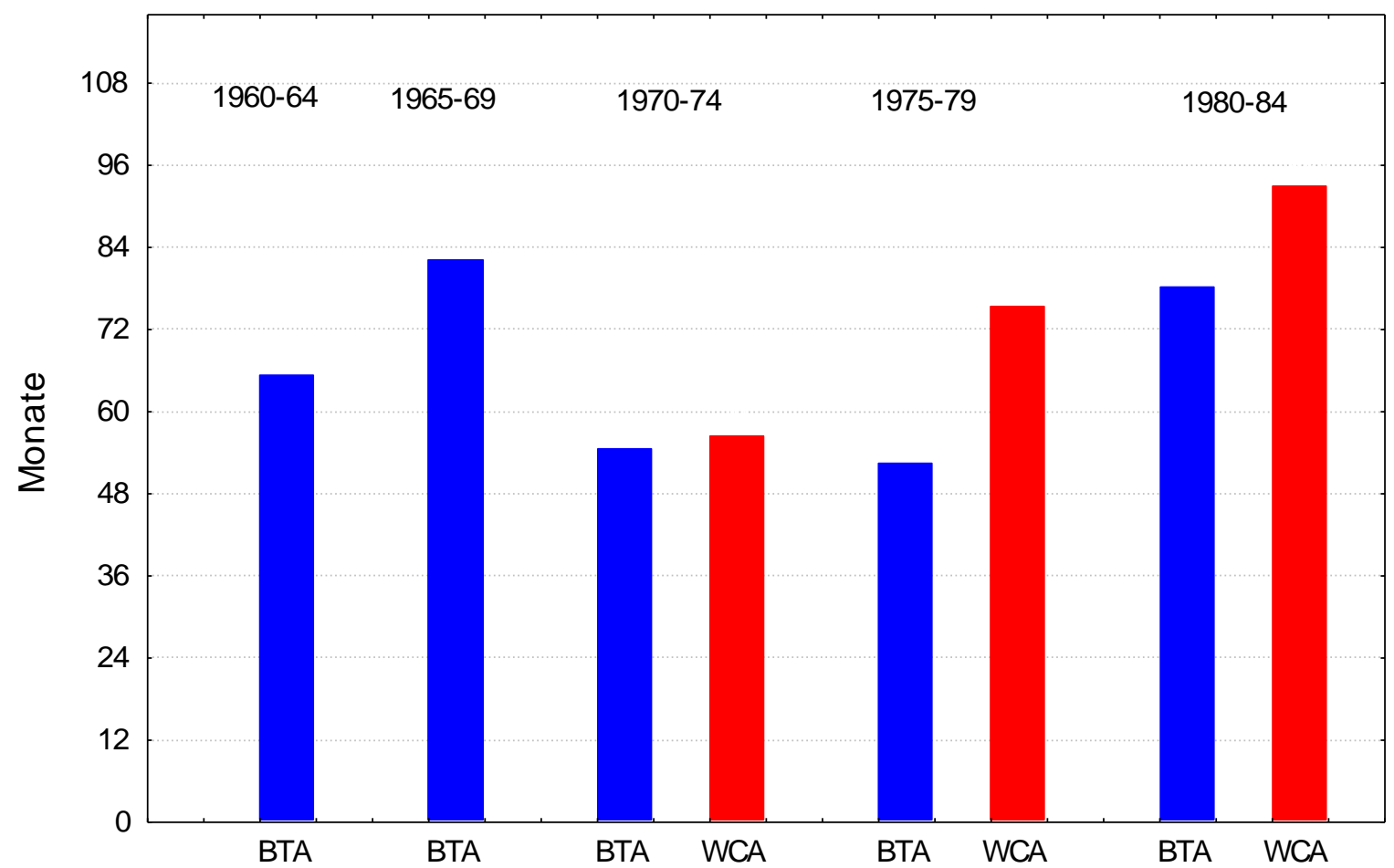

Abb. 3.7 Intervall zwischen Palliativ-OP und Korrektur-OP

\subsubsection{Intraoperative Daten bei der Korrektur-Operation}

Während der Korrektur-Operation wurde eine Kommissurotomie bei 119 Patienten (36,7\%) durchgeführt. Diese war in der BTA-Gruppe mit 96 Patienten $(38,4 \%)$ häufiger als in der anderen Gruppe (Abbildung 3.8).

Das Infundibulum wurde bei 294 Patienten $(90,7 \%)$ reseziert: In der BTA-Gruppe bei 232 Patienten ( $=92,8 \%$ ), in der WCA-Gruppe bei 48 Patienten (=84,2\%) (Abb. 3.8)

Eine Erweiterungsplastik der Ausflussbahn mittels eines Patch wurde bei 209 Patienten (64,5\%) durchgeführt. Verglichen mit der BTA-Gruppe wurde ein subvalvulärer Patch häufiger in der WCA-Gruppe implantiert $(63,2 \%$ vs. $70,2 \%$; Abbildung 3.8).

Die Erweiterungsplastik war transannulär bei 102 Patienten (48,8\%): In der BTAGruppe waren es 70 Patienten bei 158 Patienten (44,3\%) und in der WCA-Gruppe deutlich häufiger mit 30 Patienten bei 40 Patienten (75\%). 
Pulmonale Conduits wurden lediglich bei 6 Patienten (2\%) implantiert: Zwei Mal in der BTA-Gruppe (1\%), drei Mal in der WCA-Gruppe (5\%) und ein weiteres Mal in der AD-Gruppe.

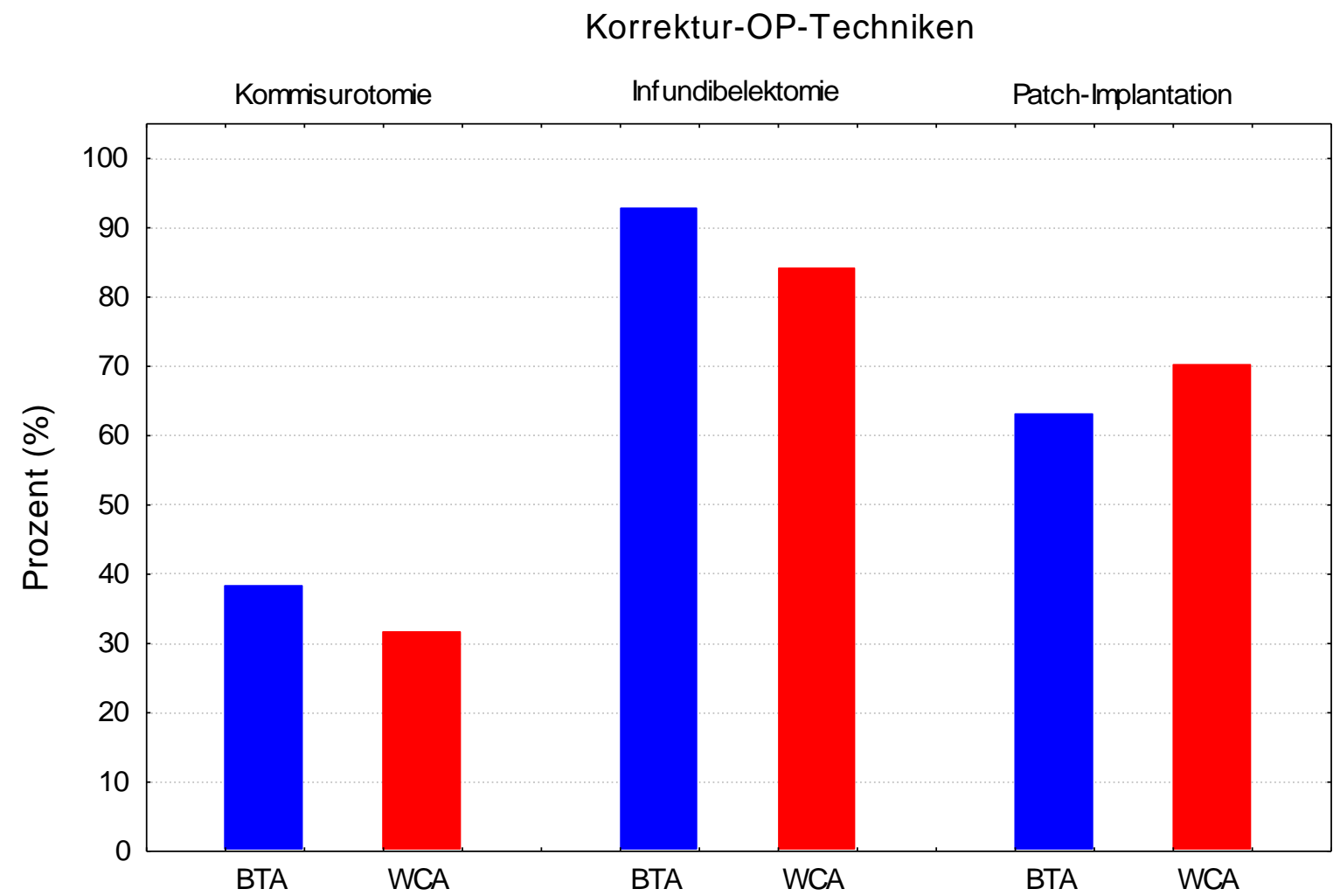

Abb. 3.8 Häufigkeiten bezüglich der Durchführung von Kommissurotomie, Infundibulektomie und Erweiterungsplastik mit Patch im Rahmen der Korrektur-Operation

Die durchschnittliche Operationsdauer aller Operationsverfahren betrug bei allen operierten Patienten $294 \pm 112$ Minuten. Die kürzeste Operationsdauer lag in der BTA-Gruppe vor mit durchschnittlich $283 \pm 105$ Minuten. Die Operationsdauer der WCA-Gruppe lag durchschnittlich bei $314 \pm 114$ Minuten und war damit deutlich länger als der Durchschnitt, jedoch nicht signifikant.

\subsection{Frühpostoperative Daten nach der Korrektur-Operation}

Nach der Korrektur-Operation verstarben 49 der 324 Patienten innerhalb der ersten 30 Tage (Frühletalität 15,1\%). Die Subgruppen-Analyse zeigt keinen relevanten Unterschied zwischen den beiden Gruppen (13,2\% in der BTA-Gruppe, 14\% in der WCA-Gruppe). 
Die Blutverluste innerhalb der ersten 48 Stunden betrugen im Mittel $973 \pm 795 \mathrm{ml}$. Die geringsten Blutverluste hatten die Patienten der WCA-Gruppe (852 $\pm 631 \mathrm{ml})$. In der BTA-Gruppe lag ein mittlerer Blutverlust innerhalb der ersten 48 Stunden von 931 $\pm 711 \mathrm{ml}$ vor. Bei 80 Patienten musste eine Rethorakotomie durchgeführt werden. Signifikant weniger Rethorakotomien wurden in der WCA-Gruppe durchgeführt (11\% vs. $25 \%$ in der BTA-Gruppe; $p=0,002$ ).

Postoperativ wurden die Patienten insgesamt $62 \pm 121$ Stunden nachbeatmet. Die Intubationsdauer war signifikant kürzer in der BTA-Gruppe (46 \pm 57 Stunden vs. 108 \pm 207 Stunden in der WCA-Gruppe; $(p=0,002)$.

Insgesamt waren die Patienten über alle Jahrzehnte für $134 \pm 135$ Stunden (5,6 Tage) auf der Intensivstation (ICU). Auf die im Vorfeld verschiedenen PalliativOperationen bezogen kam es zu einer signifikanten Verkürzung der Liegedauer auf der Intensivstation bei den Patienten, die vorher eine BTA bekommen haben (117 \pm 100 Stunden). Die Patienten der WCA-Gruppe hatten eine signifikant längere Liegedauer von $189 \pm 205$ Stunden $(p<0,001)$.

Betrachtet man die verschiedenen Jahrzehnte in 5 Jahresintervallen, so zeigen sich teilweise deutliche Abweichungen in der Liegedauer (Tabelle 3.5). Ab 1960 stieg die mittlere Liegedauer auf der ICU von $93 \pm 96$ Stunden bis auf $153 \pm 170$ Stunden Ende der '70er Jahre. Ab 1980 zeigte sich eine Reduktion der mittleren Liegedauer auf $123 \pm 81$ Stunden. Im Vergleich zwischen den beiden Gruppen hatte die BTA-Gruppe kürzere Liegezeiten als die WCA-Gruppe (Tabelle 3.5).

Tabelle 3.5 Intensivaufenthalt nach der Korrektur-Operation (in Stunden postoperativ)

\begin{tabular}{|l|l|l|l|l|}
\hline & alle Patienten & BTA-Gruppe & $\begin{array}{l}\text { WCA- } \\
\text { Gruppe }\end{array}$ & P \\
\hline Gesamtzeitraum & $134( \pm 135)$ & $117( \pm 100)$ & $189( \pm 205)$ & 0,00036 \\
\hline $1960-64$ & $93( \pm 96)$ & $93( \pm 96)$ & - & - \\
\hline $1965-69$ & $104( \pm 88)$ & $104( \pm 88)$ & - & - \\
\hline $1970-74$ & $123( \pm 99)$ & $125( \pm 104)$ & $104( \pm 44)$ & 0,52 \\
\hline $1975-79$ & $153( \pm 170)$ & $113( \pm 103)$ & $224( \pm 235)$ & 0,0008 \\
\hline $1980-84$ & $123( \pm 81)$ & $130( \pm 88)$ & $89( \pm 56)$ & 0,31 \\
\hline
\end{tabular}




\subsection{Klinische Langzeitergebnisse}

Im weiteren Follow up (156 \pm 105 Monate) sind 12 weitere Patienten verstorben $(3,7 \%)$. Davon waren 8 Patienten $(3,2 \%)$ aus der BTA-Gruppe und 3 Patienten aus der WCA-Gruppe (5,3\%).

Somit sind inklusive der Frühletalität insgesamt 61 Patienten verstorben, das entspricht $18,8 \%$ der insgesamt operierten Patienten. In der WCA-Gruppe lag die Gesamtletalität mit 19,3\% (11 Patienten) höher als in der BTA-Gruppe mit 16,4\% (41 Patienten). Besonders hoch war die Gesamtletalität in der AD-Gruppe mit 52,9\% der dort operierten Patienten (9 Patienten, s. Anhang 3).

Beim letzten Kontakt befanden sich die Patienten durchschnittlich im NYHA-Stadium 2,3 ( \pm 1.0$)$. Innerhalb der Gruppen zeigten sich keine relevanten Unterschiede. In der BTA-Gruppe lag der Mittelwert der NYHA bei 2,2 $( \pm 1,0)$ und in der WCA-Gruppe etwas höher mit einem Mittelwert von 2,7 $( \pm 0,8)$ (Abb. 3.9). 

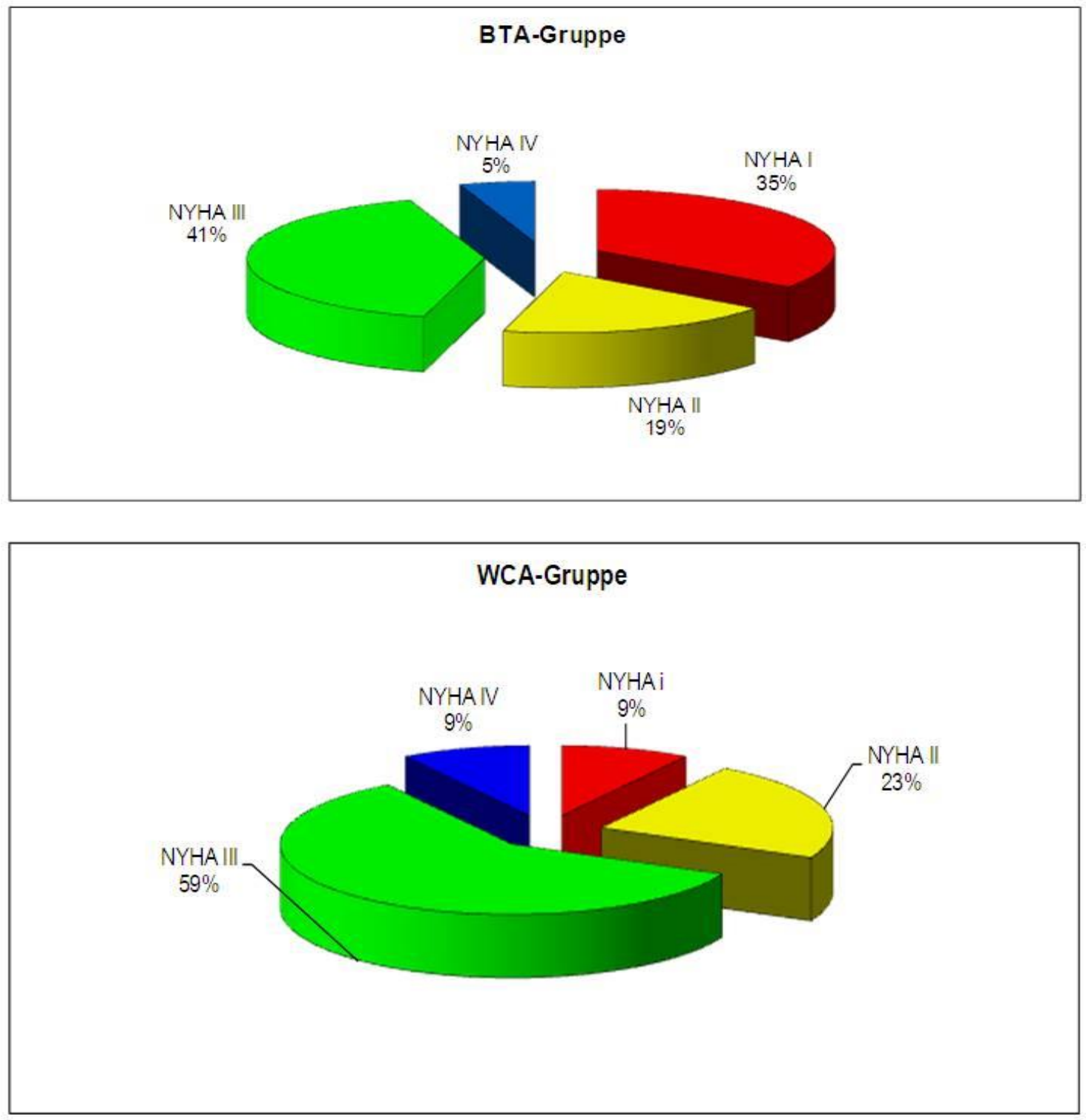

Abb. 3.9 NYHA-Stadium der Gruppen beim letzten Kontakt

\subsection{Hämodynamische Veränderungen}

Die Korrektur-Operation führte in allen Gruppen zu einer effektiven Veränderung der Ventrikeldrücke (Tabelle 3.6). 
Tabelle 3.6 Veränderung der Ventrikeldrücke (in mmHg) im Prä- und Post- OP- Vergleich.

RVP: rechtsventrikulärer Druck, LVP: linksventrikulärer Druck

\begin{tabular}{|l|l|l|l|l|}
\hline & Ventrikeldrücke & Prä-OP & Post-OP & $\mathbf{P}$ \\
\hline BTA & LVP mean & $96,5( \pm 18,28)$ & $92,6( \pm 18,51)$ & $\mathbf{0 , 0 3 4}$ \\
\hline & RVP mean & $96,01( \pm 21,17)$ & $52,75( \pm 15,79)$ & $<\mathbf{0 , 0 0 1}$ \\
\hline WCA & LVP mean & $95,43( \pm 18,16)$ & $90,31( \pm 15,45)$ & $\begin{array}{l}\text { nicht } \\
\text { signifikant }\end{array}$ \\
\hline & RVP mean & $97,78( \pm 34,73)$ & $59,05( \pm 15,04)$ & $<\mathbf{0 , 0 0 1}$ \\
\hline
\end{tabular}

Der präoperative Quotient des systolischen rechtsventrikulären Druckes zum systolischen linksventrikulären Druck lag in allen Gruppen um 1 (BTA: 0,9995 \pm 0,1661, WCA: 1,0133 \pm 0,30113). Postoperativ wurde der Quotient (RVP syst./LVP syst.) in allen Gruppen signifikant gesenkt (Abbildung 3.10).

$\mathrm{RV} / \mathrm{LV}$

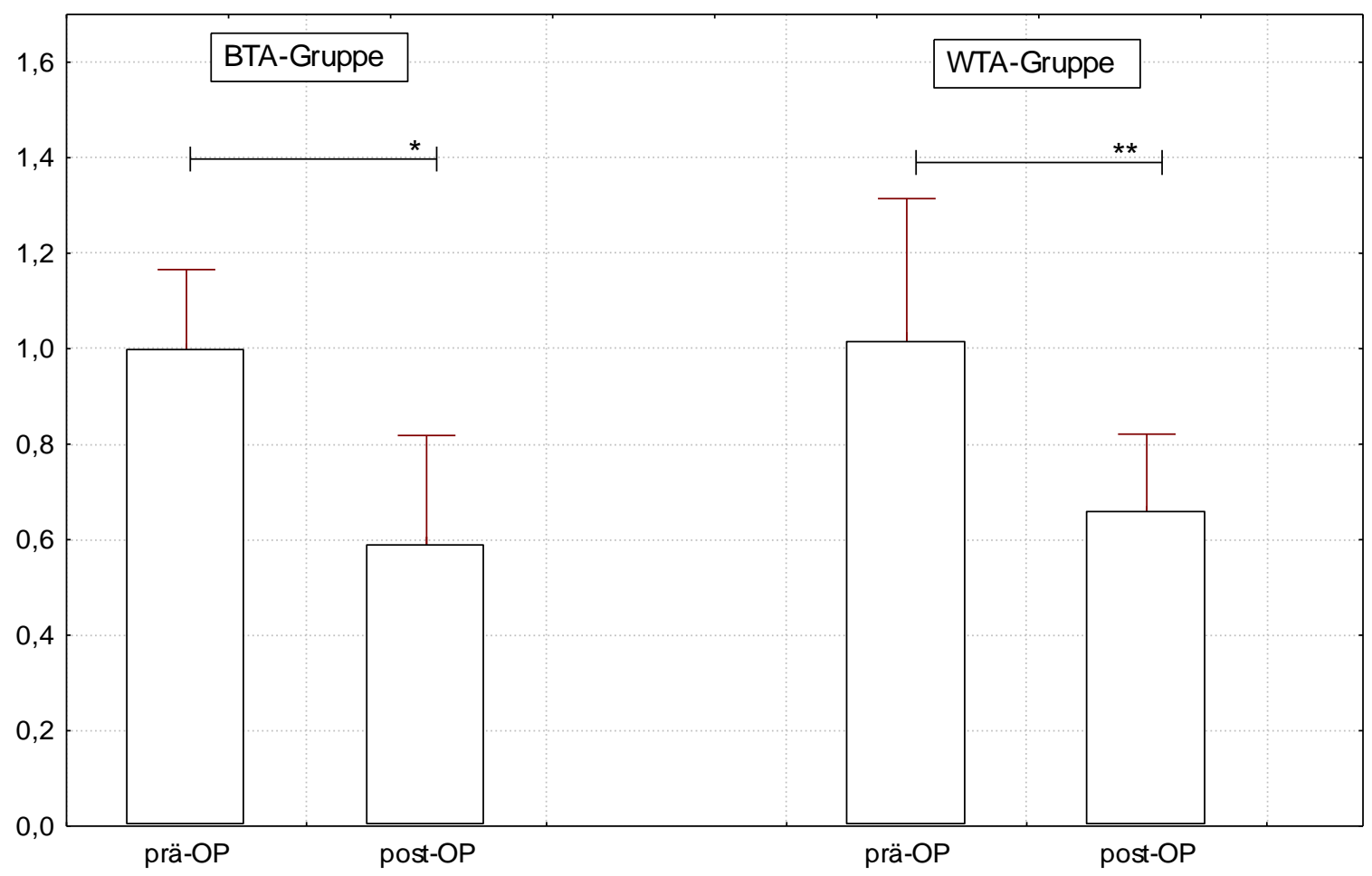

Abbildung 3.10 Veränderung des rechtsventrikulären/ linksventrikulären Druckquotienten im Vergleich zwischen prä- und postoperativ in der BTA- und WCA-Gruppe. * $p<0,00000001,{ }^{* *}: p=000001$ 


\subsection{Radiologische Veränderungen}

Der Herz-Thorax-Quotient (HTQ) betrug beim Gesamtkollektiv präoperativ im Mittel $0,56( \pm 0,07)$. In der BTA-Gruppe veränderte sich der HTQ von präoperativ 0,55 ( \pm $0,07)$ auf postoperativ $0,54( \pm 0,05)$ und in der WCA-Gruppe entsprechend von 0,58 $( \pm 0,08)$ auf $0,54( \pm 0,05)$.

\subsection{Veränderungen des Lagetyps im EKG}

Der Lagetyp wurde bei 250 Patienten (152 Patienten in der BTA-Gruppe und 36 Patienten in der WCA-Gruppe) bestimmt.

Präoperativ fand sich am häufigsten der Rechtslagetyp: In der BTA-Gruppe mit 49\%, in der WCA-Gruppe mit 47\% und in der AD-Gruppe mit 29\% (Abb. 3.11). Der zweithäufigste Lagetyp war der überdrehte Rechtstyp, mit 8\% in der BTA Gruppe und $7 \%$ in der WCA Gruppe.

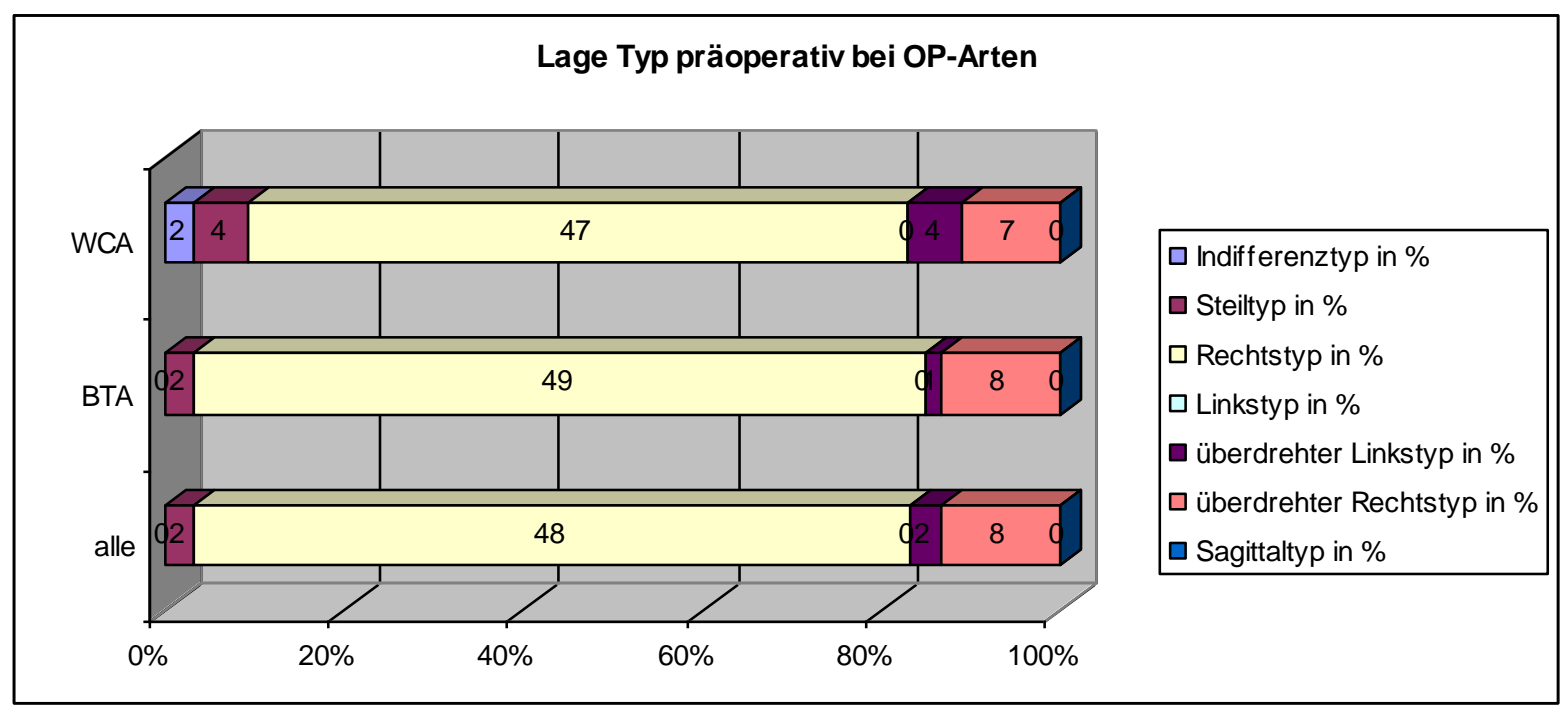

Abb 3.11 Der Lagetyp vor der Korrektur-Operation in den verschiedenen Gruppen nach der PalliativOperation

Postoperativ nahm vor allem der Anteil von Rechtslagetyp ab (Abb. 3.12): In der BTA Gruppe gab es nur noch 18\% Rechtstypen und in der WCA-Gruppe $21 \%$. In der BTA-Gruppe kam es zu einer deutlichen Erhöhung des Indifferenztyps mit 13\%, 
gefolgt vom Steiltyp mit $12 \%$ und Linkstypen mit $8 \%$. Der überdrehte Rechts- und Linkstyp lag in 5\% der Fälle vor. Der zweithäufigste Lagetyp war in der WCA-Gruppe postoperativ der Linkstyp mit 18\%, gefolgt vom überdrehten Linkstyp und Steiltyp mit jeweils $11 \%$. Der Indifferenztyp war mit einem Anteil von $4 \%$ gering.

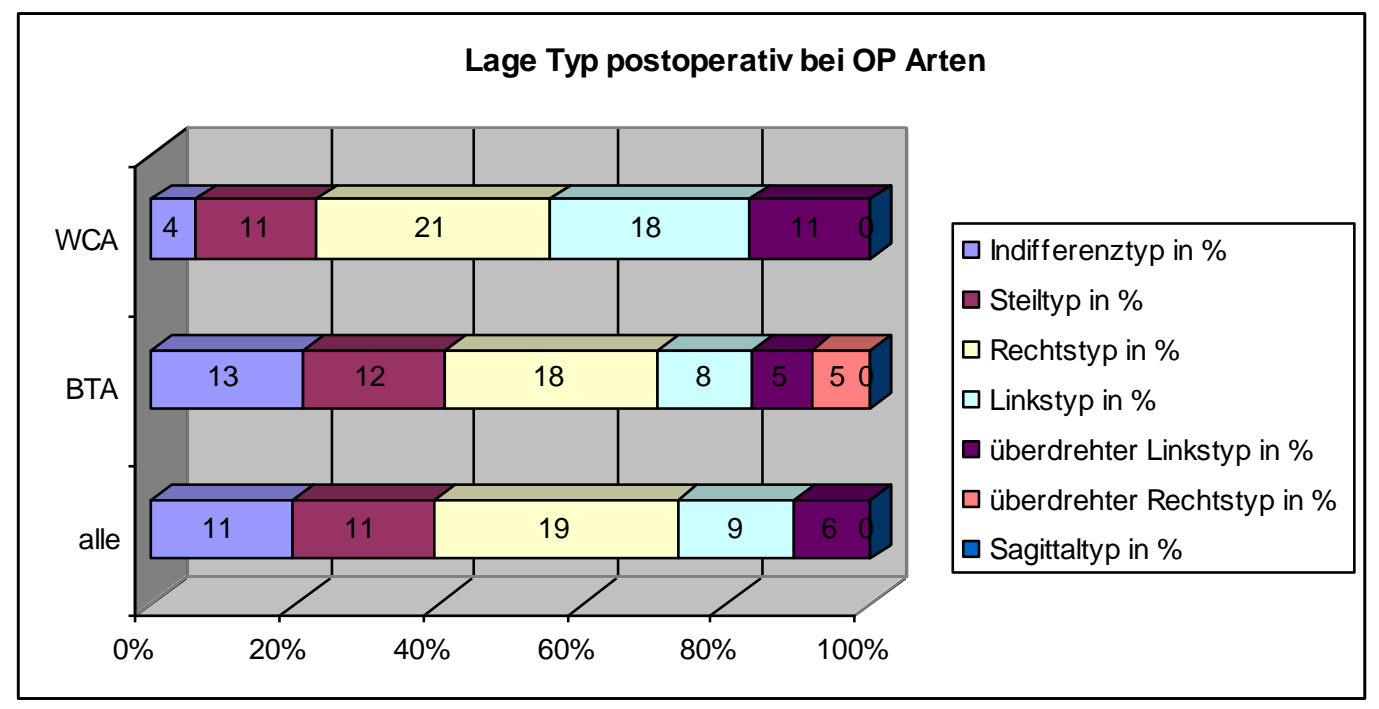

Abb. 3.12 Der Lagetyp nach der Korrektur in den verschiedenen Gruppen (gemäß der vorherigen Palliativ-Operation) 


\section{Diskussion}

Die Fallotsche Tetralogie ist mit 10\% aller kongenitalen Herzfehler der häufigste zyanotische Herzfehler (Babu-Narayan und Gatzoulis 2011). Kiran et al. 2011 hatten bei ihrer Untersuchung von pädiatrischen kardiologischen Erkrankungen eine Geschlechterverteilung von 1.6/1 (ratio male/ female) bei der Fallotschen Tetralogie gefunden. Dies erscheint im Vergleich zu unserem untersuchten Kollektiv relativ hoch. In unserem Kollektiv zeigt sich eine geringere männlich/ weiblich-Dominanz bei der Geschlechterverteilung von 1.25/1 (ratio male/ female). Ähnlich zu unseren Daten fanden Van Arsdell et al. 2000 einen diskret höheren männlichen Anteil (56\%). Eine direkte geschlechtsspezifische genetische Korrelation wurde nicht nachgewiesen. Neueste Studien zeigen aber neben der Korrelation der Fallotschen Tetralogie mit Trisomie 21 (Marino et al. 1996) einen Zusammenhang mit einer Schädigung des chromosomalen Bandes 7p22. Richards et al. 2011 konnten zeigen, dass diese Region 4 Gene kodiert (MAD1L1, FTSJ2, NUDT1 und SNX8), welche allein oder in Kombination eine wichtige Rolle bei der Entwicklung des Herzens zu spielen scheinen.

\subsection{Indikationen zur operativen Behandlung}

Eine operative Intervention ist bei der Fallotschen Tetralogie unumstritten. Bereits 25\% der Patienten sterben innerhalb des ersten Lebensjahres, wenn der Herzfehler operativ nicht angegangen wird. Und diese Prognose wird über die Jahre deutlich schlechter. Bis zum 3. Lebensjahr sterben schon $40 \%, 70 \%$ bis zum Erreichen des 10. Lebensjahres und bis zum 40. Lebensjahr sind $95 \%$ der Patienten ohne operative Intervention verstorben. Gründe für diese hohe Letalität ohne Intervention liegen in der steigenden Zyanose durch den rechts-links-Shunt auf Ventrikelebene, zunehmenden Arrhythmien, einer steigenden Thrombosetendenz und dem Entstehen von zerebralen Abszessen (Babu-Narayan und Gatzoulis 2011). Die Zyanose der Patienten ist vom Grad der rechtsventrikulären Ausflusstraktobstruktion abhängig. Lediglich bei dem sogenannten Pink Fallot mit einem geringen Grad der rechtsventrikulären Ausflusstraktobstruktion ist ein Überleben ohne Operation bis ins hohe Alter möglich. 


\subsection{Operatives Verfahren}

Sowohl die Art und Weise der operativen Versorgung der Fallotschen Tetralogie als auch der beste operative Zeitpunkt werden auch heutzutage kontrovers diskutiert. In der Literatur zeigen sich zwei Trends: Während die erste Gruppe zunächst eine Palliation bei den symptomatischen Kindern empfiehlt, um das Wachstum der Pulmonalarterien anzuregen und dann in einem zweiten Eingriff die endgültige Korrektur-Operation durchzuführen (Yuan et al. 2009), befürwortet die zweite Gruppe eine frühe Korrektur-Operation, möglichst in den ersten Lebensmonaten, und verweist auf die gute Langzeitprognose (Hirsch et al. 2000, Hashemzadeh und Hashemzadeh 2010).

In der Studie von Nollert et al. 2003 wurden vier Prädiktoren für einen plötzlichen Herztod nach Korrektur-Operation identifiziert: 1) Die Anwendung des Patches zur Erweiterung des Ausfußtraktes, 2) das männlichen Geschlecht, 3) eine höhere NYHA-Klasse vor der Korrektur-Operation und 4) eine Korrektur-Operation ohne vorherige Palliativ-Operation. Im Umkehrschluss könnte man postulieren, dass eine vorausgehende Palliation das Risiko für einen plötzlichen Herztod im Verlauf minimieren kann.

In der vorliegenden Arbeit wurden lediglich die Patienten untersucht, die eine Korrektur-Operation nach vorheriger Palliation erhalten haben.

\subsection{Klinische Befunde}

Die bei der Fallotschen Tetralogie häufig beschriebenen präoperativen klinischen Parameter wie Dyspnoe, Zyanose und hypoxische Anfälle (Bailliard und Anderson 2009) fanden sich in beiden Gruppen. Die größten Prozentzahlen der ungünstigen präoperativen klinischen Parameter zeigten sich in der Gruppeneinteilung bei der BTA-Gruppe. Das von uns untersuchte Kollektiv zeigte sehr hohe Anzahlen an hypoxischen Anfällen (12\% aller Patienten). Auffällig häufig waren hypoxische Anfälle in der WCA-Gruppe (19\%). Die hypoxischen Anfälle gelten in der Literatur (Bailliard und Anderson 2009) als ein Marker der schwersten Form der Fallotschen Tetralogie und werden in der heutigen Zeit kaum noch gefunden. Man kann davon ausgehen, dass sich vor allem in der WCA-Gruppe kränkere Kinder befanden. 
Die Erhöhung der Blutwerte für Hämoglobin und Hämatokrit sind ein Marker für die chronische Hypoxie der Kinder. Diese führt zu einer Gewebsanoxie und zu einem verminderten peripheren Blutfluss. Bei der Fallotschen Tetralogie sind diese beiden Parameter deutlich erhöht. Von deren Höhe hängen unter anderem das Risiko eines thrombembolischen Geschehens ab und damit die Sterblichkeit. Schon 1971 stellten Rygg et al. 1971 bei der Nachuntersuchung von 206 Patienten mit Palliativ-Operation die Indikation für eine Shunt-Operation anhand der Höhe des Hämoglobinwertes. Ab einem Hämoglobinwert von $20 \mathrm{~g} / \mathrm{dl}$ stellten sie eine absolute Indikation für eine Shunt-Operation, um so ein günstigeres Operationsalter für die Korrektur-Operation zu erhalten. In unserer untersuchten Patientengruppe zeigte die BTA-Gruppe die höchsten Werte mit 17,6 g/dl. Die Werte sind im Vergleich zu van Arsdell et al. 2000, die ein mittleres präoperatives Hämoglobin von $14,0 \mathrm{~g} / \mathrm{dl}$ bei ihren untersuchten Kindern hatten, deutlich höher.

\subsection{Morphologische Befunde}

Assoziierte Vitien traten in allen Gruppen annährend gleich häufig auf, lediglich in der WCA-Gruppe gab es vermehrt einen PDA mit $11 \%$ bei einem durchschnittlichen Vorkommen in allen Gruppen von 5\%. Bei der Fallotschen Tetralogie treten assoziierte Vitien in ca. $1 / 3$ der Fälle auf (Saeed et al. 2009), auch in der Studie von Cho et al. 2002 trat in 10\% ein assoziierter PDA in der Palliativ-Gruppe auf. Häufiger als einen PDA fand sich im untersuchten Kollektiv ein ASD mit im Mittel 11\% aller Patienten. Duro et al. 2010 bezifferten das durchschnittliche Vorkommen von einem ASD bei Fallotscher Tetralogie mit 2\%. Alsoufi et al. 2007 fanden, dass sich hierdurch eine Korrektur-Operation erschwert. Somit ist auch aufgrund der assoziierten Vitien von einem erschwerten operativen Management der untersuchten Kinder auszugehen.

Bei den Arten der Pulmonalstenose zeigte sich eine Häufung der valvulären Pulmonalstenosen. 


\subsection{Palliativ-Operationen}

\subsubsection{Alter bei der Palliation}

Die Patienten in der BTA-Gruppe waren zum Zeitpunkt der später durchgeführten Korrektur-Operation 8,7 $( \pm$ 4,7) Jahre alt. Die Patienten in der WCA-Gruppe wurden vergleichsweise deutlich früher korrigiert mit einem Altersdurchschnitt von $7,1( \pm 2,9)$ Jahren.

Bei Stewart et al. 1988 sind die Patienten deutlich jünger zum Zeitpunkt der Korrektur-Operation. In seiner Studie mit 85 Patienten von 1973-1986 erfolgte nach einer durchgeführten BTA- Shunt-Operation eine Korrektur-Operation im Durchschnitt nach 3,9 Jahren. Hierfür kann es mehrere Gründe geben: zum einen handelt es sich bei dem von uns untersuchten Kollektiv um Operationen seit 1960 und in den letzten Jahrzehnten hat sich das Behandlungsmanagement hin zu immer früheren Operationen gewandelt, zum anderen liegt bei seiner Studie ein deutlich kleinerer Patientenstamm und Untersuchungszeitraum vor.

Ein weiterer Beleg hierfür ist die Studie von Kanter et al. 2010, hier erfolgte nach der vorhergehenden BTA- Shunt-Operation die Korrektur-Operation im Durchschnitt nach 216 ( \pm 99 ) Tagen und damit zu einem noch früheren Zeitpunkt. Bei der Studie von Kanter et al. 2010 wurden zwischen 2002 und 200837 Patienten mit Fallotscher Tetralogie untersucht. 17 Patienten erhielten zunächst eine BTA- Shunt-Operation (meist als Notfalloperation) und 20 Patienten eine direkte Korrektur-Operation. Bei dieser Studie wurden mit 37 eingeschlossenen Patienten deutlich weniger Kinder nachuntersucht als in unserer Studie. Damit kann man die Studie als weniger repräsentativ ansehen als das von uns untersuchte Kollektiv von 324 Patienten.

\subsubsection{Zentraler vs. peripherer Shunt}

Wie bereits erwähnt, war die am häufigsten durchgeführte Palliativ-Operation in unserem untersuchten Kollektiv vor der endgültigen Korrektur-Operation die BlalockTaussig-Anastomose mit insgesamt $77,2 \%$ als peripherer Shunt, gefolgt von der Waterston-Cooley-Anastomose mit vergleichsweise wenigen Patienten (17,5\%) als zentral angelegter Shunt. Da es sich bei diesen beiden Gruppen bereits um 94,7\% der untersuchten Patienten handelt, lassen sich die Daten sehr gut als Vergleich zwischen einer zentralen Shunt-Operation und einer peripheren Shunt-Operation 
verwenden. Man sollte meinen, dass vorwiegend ein zentraler Shunt favorisiert wird, da dieser eine bessere und schnellere Ausbildung der verkümmerten Pulmonalgefässe erwarten lässt. Doch bereits Tláskal et al. 1985 beschreiben eine erhöhte Mortalität und eine prozentuell erhöhte postoperative pulmonale Hypertonie. Genauso kritisch äußern sich Stanley et al. 1981 gegenüber der Waterston-CooleyAnastomose. Sie bezeichnen sie als eine Hochrisikooperation, welche lediglich zu empfehlen ist bei symptomatischen Kindern innerhalb der ersten Lebenswochen, bei denen aufgrund der Anatomie der Arteria subclavia das Anlegen einer BlalockTaussig-Anastomose nicht möglich ist. Bei einem zentral angelegten Shunt liegt außerdem die Vermutung nahe, dass im Vergleich zu einem peripheren Shunt bei der im Verlauf folgenden Korrektur-Operation vermehrt Probleme in Form von Verwachsungen auftreten könnten. Bei unserem Patienten-Kollektiv fanden wir jedoch kein höheres Risiko bei der Korrektur nach einer WCA.

\subsubsection{Rezidiv-Eingriffe}

In insgesamt $17 \%$ der Fälle wurde vor der endgültigen Korrektur-Operation eine zweite Palliativ-Operation notwendig. Damit liegt die Re-Palliationsrate knapp über der bei Yuan et al. 2009 mit 15,4\% beschrieben Reoperationsrate. Im untersuchten Kollektiv wurde sowohl in der BTA-Gruppe als auch in der WCA-Gruppe eine BTA bevorzugt: In der BTA-Gruppe der entsprechend kontralateralen Seite, um im kontralateralen Stromgebiet eine bessere Durchblutung zu erreichen, in der WCAGruppe wurde durch eine BTA eine verbesserte Durchblutung in der Peripherie erreicht. Bei $3 \%$ der BTA-Gruppe wurde als zweiter Eingriff ein zentraler Shunt angelegt (WCA). Es scheint, als ob ein zentraler Shunt entgegen allen Erwartungen eher zu der Notwendigkeit einer zweiten Palliativ-Operation mit Anlage eines weiteren peripheren Shunts führt.

Das Zeitintervall bis zur zweiten Palliativ-Operation war bei beiden Gruppen annährend gleich mit im Durchschnitt 38,5 Monaten. Bei Yuan et al. 2009 lagen hingegen nur 12,4 bis 26,1 Monate zwischen den erneuten Operationen. In der Studie von Williams et al. 2007, die zweitausend BTA-Shunt-Operationen über sechzig Jahre nachuntersuchten, zeigte sich zwischen der ersten BTA und der zweiten BTA ein Intervall von 33,6 Monaten. Dies entspricht in etwa dem von uns ermittelten Intervall. 


\subsection{Korrektur-Operation nach vorheriger Palliation}

\subsubsection{Intervall zwischen Palliativ- und Korrektur-Operation}

Aufgrund der Entwicklung über die Jahrzehnte ist es sinnvoll, sich die Intervalle zwischen der Palliativ-Operation und der Korrektur-Operation über die Zeit anzusehen. Hierzu führten wir beim untersuchten Patientenkollektiv eine Aufschlüsselung in 5 Jahresschritten durch. Das Intervall zwischen der ersten Palliativ-Operation und der anschließenden Korrektur-Operation liegt Anfang der sechziger Jahre bei 67,7 Monaten. In der "frühen Zeit“ der operativen Versorgung der Fallotschen Tetralogie ist eine längere Spanne zwischen der Palliation und der anschließenden Korrektur-Operation keine Ausnahme, wie bereits oben erwähnt. Aufgrund der eingeschränkten technischen Unterstützungsmöglichkeiten bei der Operation Anfang der sechziger Jahre und dem instabilen Patientenkollektiv wurde zunächst ein vergleichsweise kleiner Palliativeingriff durchgeführt und das Kind stabilisiert, bevor eine Korrektur-Operation erfolgte. Über die Jahre steigt das Intervall zunächst an, bevor es Anfang der siebziger Jahre deutlich sinkt auf einen Durchschnittswert von 55 Monaten. Dies entspricht der heutigen Vorgehensweise, eine frühe Korrektur des Herzfehlers wird bevorzugt. Bei der bereits oben erwähnten Studie von Kanter et al. 2010 liegen zwischen der Palliativ-Operation und der Korrektur-Operation sogar nur 7 Monate, dies entspricht in etwa auch den anderen neueren Literaturangaben. Beim untersuchten Kollektiv stieg das Intervall zwischen den beiden Operationen in der darauf folgenden Zeit von 1980-1984 entgegen den Erwartungen wieder bis auf 83 Monate an. Die Gründe hierfür sind nicht klar, zwischen den Gruppen zeigten sich jedoch keine signifikanten Unterschiede. Das Intervall schien also nicht von der vorher durchgeführten Palliativ-Operation abzuhängen.

\subsubsection{Operationstechniken der anschließenden Korrektur-Operation}

Am häufigsten wurde eine Infundibulektomie bei allen Patienten durchgeführt (durchschnittlich 90,7\%). Signifikante Unterschiede traten dabei nicht innerhalb der 
Gruppen auf. Rechtsventrikuläre Ausflusstrakterweiterungen in Form einer Patchimplantation wurden am zweithäufigsten durchgeführt mit durchschnittlich 60,5\%. Hier zeigten sich ebenfalls keine signifikanten Unterschiede zwischen den Gruppen. Nur bei der genaueren Differenzierung in transannuläre und nicht transannuläre Patches kam es zu einer deutlichen Unterscheidung mit $75 \%$ transannulären Patches in der WCA-Gruppe und $44,3 \%$ in der BTA-Gruppe. Warum ist dies so wichtig? Bereits in der Literatur liegen mehrere Studien über transannuläre Patches bei Fallotscher Tetralogie vor. Sie haben zwar keine erhöhte Sterberate erzeugt, aber eine signifikant erhöhte Rate an Reoperationen (Lindberg et al. 2011). Van Arsdell et al. 2000 zeigten in ihrer Studie, dass die Kinder mit einem transannulären Patch eine verlängerte Beatmungszeit (und damit verbundene verlängerte Intensivaufenthaltszeit) sowie eine erhöhte Pulmonalinsuffizienzrate aufweisen. Transannuläre Patches sind damit als ungünstig zu bewerten. In einer Studie aus Paris wurden 56 Patienten mit Fallotscher Tetralogie mit einem Alter unter 6 Monaten nachuntersucht (Sousa et al. 1994). Hier zeigte sich eine signifikant niedrigere Inzidenz für einen transannulären Patch bei Patienten, welche zuvor eine Palliation erhalten hatten ( $9 \%$ transannuläre Patches) im Vergleich zu der Gruppe mit einer direkten Korrektur-Operation mit 56\% transannulären Patches. Auch dies sollte in die Beurteilung des optimalen Therapieverfahrens der symptomatischen Kinder mit Fallotscher Tetralogie mit einfließen.

Am dritthäufigsten wurde eine Komissurotomie durchgeführt. Conduit-Operationen wurden extrem selten, mit durchschnittlich $2 \%$ der Operationen, durchgeführt. Signifikante Unterschiede innerhalb der Gruppen traten nicht auf.

\subsubsection{Operationsdauer bei der Korrektur-Operation}

Die Operationsdauer stellt einen indirekten Marker für die Schwere der Operation dar. Diese ist von unterschiedlichen Aspekten abhängig. Einerseits von der Schwere des Herzfehlers, andererseits aber auch von den Verwachsungen durch die bereits durchgeführte Palliativ-Operation. Unter Berücksichtigung dieses Aspektes ist der Vergleich zwischen den unterschiedlichen Gruppen sehr interessant. In dem untersuchten Kollektiv führte ein vorangegangener zentraler Shunt wie bei einer WCA zu einer deutlich verlängerten Operationsdauer, was auf Verwachsungen im 
Operationsgebiet zurückgeführt werden könnte. Der periphere Shunt (BTA) zeigt hingegen eine deutlich verkürzte Operationsdauer.

\subsection{Frühletalität und -morbidität nach „,gestufter“ Korrektur}

In unserem untersuchten Patientenkollektiv betrug die mittlere Frühletalität 15,1\%. Cho et al. 2002 untersuchten 495 Patienten mit Fallotscher Tetralogie, die zwischen 1977 und 1999 operiert wurden und teilten diese in zwei Gruppen: In der ersten Gruppe wurden ausschließlich Palliativ-Eingriffe durchgeführt (Potts-Anastomose, WCA, BTA, etc.) und in der zweiten Gruppe eine Korrektur-Operation entweder primär oder nach vorheriger Palliativ-Operation. Während in der ersten Gruppe mit nur Palliativeingriffen die Frühletalität $16,3 \%$ betrug war diese nach den Korrektureingriffen deutlich niedriger (7,8\%). Ein Vergleich zu unseren Daten fällt jedoch schwer aus zwei Gründen: einerseits sind die Untersuchungszeiträume unterschiedlich und anderseits sind in der für uns relevanten zweiten Gruppe die Patienten sowohl primär als auch sekundär einer Korrektur-Operation unterzogen worden. Auch wenn man die Literaturdaten bezüglich der Ergebnisse nach Palliativeingriffen analysiert, stellt man fest, dass die Letalität über die letzten Jahrzehnte abgenommen hat (Williams et al. 2007). Dies liegt einerseits an der verbesserten Operationstechnik an sich und anderseits an der besseren postoperativen Versorgung inklusive apparativer Ausstattung.

Insgesamt kann man somit bei unseren Daten von einem guten postoperativen Ergebnis sprechen, insbesondere wenn man beachtet, dass es sich bei den untersuchten Kindern um ausgeprägte Formen der Fallotschen Tetralogie handelt. Innerhalb der beiden Behandlungsgruppen zeigten sich keine signifikanten Unterschiede.

Bei den postoperativen Intensivdaten (Intubationsdauer, Blutverlust in 48 Stunden und Rethorakotomierate) zeigte sich ein Trend zu Gunsten der BTA-Gruppe. Die BTA-Gruppe hatte eine signifikant kürzere Liegedauer auf der Intensivstation sowie eine signifikant kürzere Beatmungsdauer. Geringeren Blutverlust und weniger Rethorakotomien hatte hingegen die WCA-Gruppe. Kanter et al. 2010 wies in seiner Vergleichsstudie zwischen direkter Korrektur-Operation und Palliation eine unterschiedlich lange Intensivdauer der Patienten nach. Er konnte zeigen, dass 
Patienten mit einer Palliation eine deutlich kürzere Intensivdauer und Krankenhausaufenthalt haben als die Kinder, die eine primäre Korrektur-Operation erhielten. Allerdings glichen sich die Aufenthaltsdaten mit der darauf folgenden KorrekturOperation an. Dennoch ist nicht außer Acht zu lassen, dass das Risiko bei vergleichsweise kleinen, gestaffelten Eingriffen deutlich niedriger ist, wie auch Cho et al. 2002 in ihrer Studie betonten.

Betrachtet man die Intensivdauer in 5-Jahres-Schritten, kann man den Verlauf über die Jahrzehnte feststellen. In den 1960er Jahren kommt es zu einem geringen Anstieg der Liegedauer und Anfang der 70er Jahre kommt es zu einem weiteren Anstieg. Nur in der BTA-Gruppe sinkt von 1975-1979 die Liegedauer auf der Intensivstation. Zu Beginn der 1980er sinkt die Intensivdauer in der WCA-Gruppe deutlich, während sie in der BTA-Gruppe wieder leicht ansteigt. Zu beachten ist jedoch, dass in der WCA-Gruppe in dieser Zeit lediglich 5 Patienten operiert wurden.

\subsection{Langzeitergebnisse}

\subsubsection{Follow up}

Das Follow up der vorliegenden Arbeit ist mit $156 \pm 105$ Monaten vergleichsweise lang. Bei ähnlichen retrospektiven Studien ist das Follow up kürzer, z.B. bei Cho et al. 2002 beträgt es im Vergleich 96 Monate und bei Hirsch et al. 2000 lediglich 62 Monate.

\subsubsection{Gesamtletalität}

Zwölf weitere Patienten $(3,7 \%)$ sind während des Follow up im weiteren Verlauf verstorben. Innerhalb der Gruppen zeigten sich keine signifikanten Unterschiede, wobei die niedrigste Prozentzahl in der BTA-Gruppe mit 3.2\% vorliegt. Die WCAGruppe zeigte eine höhere Spätletalität von 5,3\%. Dies ist ein sehr gutes Ergebnis und entspricht dem in der Literatur beschriebenen Verlauf, dass die meisten Patienten früh-postoperativ versterben im Rahmen der bereits bestehenden Dekompensation, welche sich nicht mehr operativ umkehren lässt.

Die Gesamtletalität des von uns untersuchten Patientenkollektivs beträgt somit 18,8\%. Im Vergleich zwischen den Gruppen zeigt die WCA-Gruppe eine gering 
höhere Gesamtletalität (19,3\% vs. 16,4\% BTA-Gruppe). Vergleichbare Daten liefern Cho et al. 2002 mit einer Gesamtletalität von 16,3\%.

\subsubsection{Funktioneller Status}

Der klinische Status zum letzten Untersuchungszeitpunkt wurde mit Hilfe der NYHAKlassifikation objektiviert. Die durchschnittliche NYHA-Klasse von 2,3 zeigt eine gute Belastbarkeit im Langzeitverlauf der operierten Kinder und ist als ein sehr gutes Langzeitergebnis zu werten. In einer Studie von Nollert et al. 2003 wurde eine Assoziation zwischen dem NYHA-Status und der Inzidenz für einen plötzlichen Herztod beobachtet: Ein plötzlicher Herztod wurde bei Patienten in den NYHAKlassen I und II nicht gesehen während in der NYHA-Klasse IV eine Häufigkeit von $14 \%$ beobachtet wurde. Hypothetisch kann es bei einer höheren Klassifikation zu einer schweren Hypoxie im hypertrophierten Herz kommen, welche zu mikroskopischen myokardialen Infarkten führen kann, die ein Substrat für Arrhythmien darstellen können (Nollert et al. 2003).

\subsection{Verlaufsanalyse und Risikofaktoren}

\subsubsection{Hämodynamik}

In allen Gruppen zeigt sich eine signifikante Drucksenkung im linken und vor allem im rechten Ventrikel.

Bei der Druckrelation zwischen dem links- und dem rechtsventrikulären Druck lassen sich sehr gut die postoperativen Drucksenkungen darstellen. Da postoperativ zur Herzentlastung vor allem eine rechtsventrikuläre Drucksenkung angestrebt wird, ist es erstrebenswert, den Nenner des Quotienten möglichst klein zu halten. Bei den erhobenen Daten der Kinder in den verschiedenen Gruppen zeigt sich ein durchweg gelungenes postoperatives Ergebnis mit einer in allen Gruppen signifikanten Drucksenkung. Dies betrifft sowohl den linken als auch den rechten Ventrikel, wobei die Druckentlastung des rechten Ventrikels in allen Gruppen deutlich besser ist. Dies wirkt sich positiv auf den Druckquotienten aus. In allen Gruppen lag präoperativ ein Druckquotient um 1 vor. Postoperativ zeigt sich eine deutliche Senkung des Quotienten auf Werte um 0,6. Betrachtet man die Beschreibungen der anderen 
Autoren in ihren Untersuchungsreihen, so stellt man fest, dass es sich auch im Vergleich zu anderen Studien um sehr gute postoperative Drucksenkungen in allen Gruppen handelt. In der bereits im Vorfeld erwähnten Langzeitstudie über die chirurgische Behandlung der Fallotschen Tetralogie von Cho et al. 2002 beschreibt der Autor in der Gruppe der Patienten, welche eine „Multi Stage“- Korrektur erhalten haben, einen postoperativen Druckquotienten von $0,66 \pm 0,18$. Es handelt sich dabei um einen Patientenpool von 168 Patienten, welche insgesamt 198 pulmonale Shunts im Vorfeld angelegt bekommen haben. Eine Differenzierung des Druckquotienten der einzelnen Shunt-Gruppen führten sie nicht durch, aber bei 123 Patienten wurden BTA-Shunts angelegt. Insgesamt kann man anhand unserer Ergebnisse sagen, dass die beste Senkung des Druckquotienten in der BTA-Gruppe erreicht wurde und damit eine bevorzugte Durchführung dieser Palliativ-Operation durchaus gerechtfertigt ist.

\subsubsection{Veränderungen im EKG}

Präoperativ zeigte sich bei allen Gruppen im EKG ein erhöhter Anteil an Rechtslagetypen im EKG (48\%), was sich durch die vermehrte Rechtsherzbelastung und einer damit verbundenen Rechtsherzhypertrophie erklären lässt. Erwartungsgemäß kam es postoperativ zu einem Lagetypwechsel in Richtung der Normaltypen: Indifferenztyp und Steiltyp (22\%).

\subsubsection{Bedeutung des Herz-Thorax-Quotienten (HTQ)}

Der Herzthoraxquotient gibt die Herzgröße im Vergleich zum Thorax an, er ist somit ein guter Marker für eine Herzhypertrophie bedingt durch Volumenbelastung. Bei der Fallotschen Tetralogie handelt es sich um einen kindlichen Herzfehler mit einer deutlich vermehrten Rechtsherzbelastung. In diesem Rahmen kommt es zu einem vergrößerten HTQ der kleinen Patienten. Je nach Dauer der Volumenbelastung ohne Palliativ- oder Korrektur-Operation steigt der HTQ weiter an. Daher kann man rückwirkend anhand der HTQ Größe auf die bereits stattgefundene Herzbelastung schließen. In unserem Patientenkollektiv zeigt sich bei allen Patientengruppen die erwartete HTQ-Vergrößerung im Vergleich zur Normalbevölkerung. Innerhalb der 
Gruppen lagen jedoch große Unterschiede vor. Während der HTQ in der BTAGruppe präoperativ am niedrigsten war $(0,55 \pm 0,07)$, lag der größte $H T Q$ in der sonst nicht analysierten AD-Gruppe vor $(0,63 \pm 0,06$, s. Anhang 2). Somit waren die Patienten in der AD-Gruppe am stärksten volumenbelastet. Bei dem postoperativen HTQ erwartet man nach einer erfolgreichen Operation ein Absinken der Werte als ein Zeichen der Herzentlastung. Dies lässt sich in der BTA- und der WCA- Gruppe erwartungsgemäß nachvollziehen, wobei der HTQ in der WCA- Gruppe um 0,04 sinkt und in der BTA-Gruppe um 0,01. In der AD-Gruppe kommt es entgegen den Erwartungen zu einem geringen Anstieg des HTQ um 0,01. Offensichtlich lag in dieser Gruppe bereits eine so ausgeprägte Rechtsherzbelastung vor, dass ein operativer Eingriff keine Besserung mehr erzielen konnte. Dabei kommt die Frage auf, an welcher Stelle der "Point of no return" vorliegt. Anhand der erhobenen Messungen in dem untersuchten Kollektiv scheint dieser bei 0,58 zu liegen. Dies könnte für zukünftige Operationen von Bedeutung sein, da man bereits vor dem entsprechenden Eingriff einen adäquaten Wert hätte, welcher die Erfolgsaussichten einer klinischen postoperativen Besserung objektiviert. Weitergehende Studien zu diesem Thema wären durchaus erstrebenswert.

\subsection{Bedeutung der vorliegenden Arbeit und Aussicht auf die Zukunft}

Über die letzten Jahrzehnte ist aufgrund der operativen Möglichkeiten der Anteil an Erwachsenen mit einem zyanotischen Herzfehler, wie der Fallotschen Tetralogie, deutlich gestiegen. Die Patienten haben auch im höheren Alter eine gute Lebensqualität, so dass eine rückwirkende Betrachtung der optimalen Therapie-verfahren durchaus sinnvoll ist, um eine weitere Optimierung der Therapie zu ermöglichen. Die Therapiemöglichkeiten sind vielfältig und auch heute noch besteht keine Einigkeit bezüglich eines Goldstandards. Da es sich bei unserem untersuchten Patientenkollektiv um eine umfangreiche Studie mit 324 Patienten handelt, welche über eine Zeitspanne von mehreren Jahrzehnten operiert wurden, sind wir in der Lage, die Entwicklung der operativen Therapie vor allem der klinisch symptomatischen Kinder widerzuspiegeln. Hierbei beschränkten wir uns auf die symptomatischen Kinder, welche eine Palliativ-Operation mit einer anschließenden Korrektur-Operation erhielten. Bei dem betrachteten Kollektiv handelt es sich um Patienten mit Fallotscher Tetralogie, die in den Jahren 1960-1984 an der 
Universitätsklinik Göttingen behandelt worden sind. Zu diesem Zeitpunkt stellte die Palliativ-Operation eine häufig notwendige Therapieoption der instabilen Patienten dar. Aufgrund des diagnostischen und technischen Fortschritts ist es heutzutage möglich, auch bei instabileren Patienten eine frühzeitige Korrektur-Operation risikoärmer durchzuführen. Im Vergleich zu im Vorfeld publizierten Analysen wurde in der hier vorliegenden Arbeit ein erheblich größeres Patientenkollektiv untersucht, was eine präzisere Beurteilung der Palliativverfahren bei Fallotscher Tetralogie ermöglicht (Cho et al. 2002, Kanter et al. 2010, Williams et al. 2007).

Anhand unserer Studie hat sich die BTA als vorteilhaftere Palliativ-OperationsVariante herausgestellt. Das resultierte unter anderem aus der kürzeren OP-Dauer, den wenigsten Patchimplantationen und der signifikant kürzeren Beatmungszeit und Intensivaufenthaltsdauer. Die BTA-Gruppe zeichnete sich zusätzlich durch die effizienteste Verbesserung der hämodynamischen Parameter, die niedrigste Frühund Spätletalität sowie insgesamt ein gutes klinisches Langzeitergebnis aus.

Retrospektiv betrachtet hängt das optimale Operationsverfahren von mehreren Faktoren ab: Alter des Patienten, klinischer Zustand und anatomische Gegebenheiten. Es sollte eine individuelle Entscheidung von Fall zu Fall erfolgen und bei notwendiger Palliativ-Operation die BTA bevorzugt werden. 


\section{Zusammenfassung}

Bei der Fallotschen Tetralogie handelt es sich um eine der häufigsten kardialen angeborenen Missbildungen. Charakterisiert ist die Fallotsche Tetralogie durch vier morphologische Korrelate: eine Pulmonalstenose, einen Ventrikelseptumdefekt, eine ante- und dextroponierte Aortenwurzel und eine Hypertrophie des rechten Ventrikels. Die Klinik ist vor allem aufgrund eines Rechts-Links-Shunts durch eine schwere Zyanose gekennzeichnet. Die Mortalität beträgt ohne eine Korrektur-Operation $70 \%$ bis zum zehnten Lebensjahr.

Es bestehen zwei Operationsmöglichkeiten, die auch heutzutage noch kontrovers diskutiert werden. Erstens- bei symptomatischen Patienten- die Durchführung einer Palliativ-Operation zur Anregung der Pulmonalgefäße und in einem zweiten Eingriff das Durchführen einer endgültigen Korrektur-Operation („gestuftes Vorgehen“) und zweitens eine Primär-Korrektur-Operation.

Diese Arbeit beschäftigt sich mit den Langzeitergebnissen von Patienten, die im Zeitraum von 1960-1984 in der Klinik für Thorax-Herz-Gefäßchirurgie in Göttingen zuerst palliativ operiert wurden und anschließend eine Korrektur des Herzfehlers erhalten haben. Die prä-, intra- und postoperativen Daten von insgesamt 324 Patienten, welche in diesem Zeitraum operiert wurden, wurden in einer retrospektiven Analyse ausgewertet. In diesem Zeitraum wurden anhand der ersten Palliativ-Operation drei Gruppen gebildet: die Blalock-Taussig-Anastomosen-Gruppe (BTA-Gruppe, $n=250$ ), die Waterston-Cooley-Anastomosen-Gruppe (WCA-Gruppe, $\mathrm{n}=57$ ) und eine dritte Gruppe aus verschiedenen Palliativ-Operationen (AD-Gruppe, $\mathrm{n}=17$ ). Bei der dritten kleinen Gruppe konnte aufgrund der extremen Inhomogenität eine weitere Subgruppenanalyse nicht erfolgen.

Bei den präoperativen Daten fanden sich keine signifikanten Unterschiede. Vor der Korrekturoperation erfolgte bei $17 \%$ eine zweite und bei $1 \%$ eine dritte PalliativOperation.

Das mittlere Alter war zur Korrektur-Operation in der BTA-Gruppe höher $(8,45 \pm 4,62$ Jahre vs. 6,89 $\pm 2,96$ Jahre in der WCA-Gruppe; $p=0,0015)$. Die Operationsdauer war in der BTA-Gruppe mit $283 \pm 105$ Minuten tendenziell kürzer im Vergleich zur WCA-Gruppe $314 \pm 114$ Minuten ( $p=0,32)$. Intraoperativ wurde unter anderem eine Erweiterungsplastik mit Patch bei 64,5\% durchgeführt (BTA: 63,2\%, WCA: 70,2\%). Von diesen Patches waren 48,8\% transannulär (BTA: 44,3\%, WCA: 75\%). 
Die Frühletalität betrug 15,1\%. Eine Rethorakotomie war nach der KorrekturOperation in der WCA-Gruppe seltener ( $11 \%$ vs. $25 \%$ in der BTA-Gruppe; $p=0,002$ ). Die Korrektur-Operation führte in allen Gruppen zu einer effektiven, signifikanten Reduktion des rechtsventrikulären Druckes (BTA: von 96,01 $\pm 21,17 \mathrm{mmHg}$ auf 52,75 $\pm 15,79 \mathrm{mmHg}$, WCA: von $97,78 \pm 34,73 \mathrm{mmHg}$ auf $59,05 \pm 15,04 \mathrm{mmHg} ; \mathrm{p}<0,001$ ). Eine mechanische Ventilation nach der Korrektur-Operation war in der BTA-Gruppe signifikant kürzer als in der WCA-Gruppe (46 \pm 57 Stunden vs. $108 \pm 207$ Stunden; $p=0,002)$, Ebenso war der Intensivaufenthalt in der BTA-Gruppe signifikant kürzer (117 \pm 100 Stunden vs. $189 \pm 205$ Stunden in der WCA-Gruppe; $p<0,001$ ). Während des Follow up starben 12 weitere Patienten, so dass die Gesamtletalität 18,8\% beträgt. Beim letzten Kontakt befanden sich die meisten Patienten im NYHA-Stadium II und III (NYHA-Stadium des Gesamtkollektivs: 2,3 $\pm 1,0$; BTA-Gruppe: 2,2 $\pm 1,0$; WCA-Gruppe: $2,7 \pm 0,8)$.

Die vorliegende Datenanalyse zeigt, dass die BTA die vorteilhaftere PalliativOperations-Variante ist. Bei der Korrektur-Operation haben die Patienten nach vorheriger BTA-Shunt-Operation tendenziell eine kürzere OP-Dauer, weniger Patchimplantationen sowie eine signifikant kürzere Beatmungs- und Intensivaufenthaltsdauer. Nach Korrektur der Fallotschen Tetralogie zeigen die Patienten eine effiziente Verbesserung der hämodynamischen Parameter und ein gutes klinisches Langzeitergebnis. Da das optimale Operationsverfahren von mehreren Faktoren abhängt, u.a. Alter des Patienten, klinischer Zustand und anatomische Gegebenheiten, muss eine individuelle Entscheidung von Fall zu Fall erfolgen. Falls eine Palliativ-Operation notwendig ist, sollte die BTA bevorzugt werden. 


\section{Anhang}

Anhang 1: Präoperative Daten der „Anderen“ Gruppe

\begin{tabular}{|c|c|c|}
\hline \multicolumn{3}{|c|}{ „Andere“ Gruppe (n=17) } \\
\hline \multicolumn{3}{|c|}{ Präoperative Daten } \\
\hline Symptome & $\mathrm{N}$ & $\%$ \\
\hline Dyspnoe & 6 & 35,3 \\
\hline Zyanose & 11 & 64,7 \\
\hline hypoxische Anfälle & 1 & 6 \\
\hline $\mathrm{Hb}(\mathrm{g} / \mathrm{dl})$ & \multicolumn{2}{|c|}{$15,43( \pm 2,8)$} \\
\hline Hkt (\%) & \multicolumn{2}{|c|}{$41,6( \pm 7,2)$} \\
\hline assoziierte Vitien & $\mathrm{N}$ & $\%$ \\
\hline PDA & 1 & 6 \\
\hline ASD & 0 & 0 \\
\hline Pulmonalstenose & $\mathrm{N}$ & $\%$ \\
\hline valvulär & 2 & 11,8 \\
\hline subvalvulär & 5 & 29,4 \\
\hline valvulär und subvalvulär & 0 & 0 \\
\hline $\begin{array}{c}\text { 3-fach (sub-, supra- und } \\
\text { valvulär) }\end{array}$ & 1 & 5,9 \\
\hline Atresie & 0 & 0 \\
\hline keine & 1 & 5,9 \\
\hline keine Angaben & 8 & 47,1 \\
\hline 2. Palliativ-Operation & & \\
\hline
\end{tabular}


Anhang 2: Daten zur Korrektur-Operation der „Anderen“ Gruppe

\begin{tabular}{|c|c|c|}
\hline \multicolumn{3}{|c|}{ Korrektur- OP } \\
\hline Altersdurchschnitt bei & \multirow{2}{*}{\multicolumn{2}{|c|}{$12,4( \pm 7,9)$}} \\
\hline Korrektur-OP in Jahren & & \\
\hline Korrektur-OP Dauer & \multicolumn{2}{|c|}{405} \\
\hline Korrektur-OP Techniken & $\mathrm{N}$ & $\%$ \\
\hline Komissurotomie & 5 & 29,4 \\
\hline Infundibulektomie & 14 & 82,3 \\
\hline Patchimplantation & 11 & 64,7 \\
\hline - davon transannulär & 2 & 18 \\
\hline pulmonale Conduits & 1 & 6 \\
\hline Verlauf Hämodynamik & Prä OP & Post OP \\
\hline $\mathrm{LV}(\mathrm{mmHg})$ & $105,69( \pm 22,31)$ & $94,31( \pm 20,56)$ \\
\hline $\mathrm{RV}(\mathrm{mmHg})$ & $109,2( \pm 27,64)$ & $53,03( \pm 30,21)$ \\
\hline Drucksenkung (RV/LV) & \multicolumn{2}{|c|}{$p=0,003$} \\
\hline \multirow[t]{2}{*}{ HTQ } & Prä OP & Post OP \\
\hline & $0,63( \pm 0,06)$ & $0,64( \pm 0,08)$ \\
\hline Lagetyp & Prä OP in \% & Post OP in \% \\
\hline Rechtstyp & 29 & 24 \\
\hline überdrehter Rechtstyp & 6 & 0 \\
\hline Steiltyp & 6 & 12 \\
\hline Indifferenztyp & 0 & 6 \\
\hline
\end{tabular}


Anhang 3: Postoperative Daten und Follow up der „Anderen“ Gruppe

\begin{tabular}{|c|c|c|}
\hline \multicolumn{2}{|c|}{ Postoperative Daten und Follow up } \\
\hline -mittlerer Zeitpunkt (h) & \multicolumn{2}{|c|}{$2160( \pm 1556)$} \\
\hline Blutverlust in 48h in (ml) & \multicolumn{2}{|c|}{35} \\
\hline Rethorakotomierate (\%) & \multicolumn{2}{|c|}{$192( \pm 213)$} \\
\hline Intubationsdauer (h) & $\mathrm{N}$ & $\%$ \\
\hline Intensivaufenthalt (h) & 8 & 47,1 \\
\hline Frühletalität & $\mathrm{N}$ & $\%, 9$ \\
\hline Spätletalität & 1 & \\
\hline Letzter Kontakt (Monate) & & $122( \pm 97)$ \\
\hline NYHA-Klasse & \multicolumn{2}{|c|}{$1,0( \pm 1,2)$} \\
\hline
\end{tabular}




\section{Literaturverzeichnis}

Alsoufi B, Williams WG, Hua Z, Cai S, Karamlou T, Chan CC, Coles JG, Van Arsdell GS, Caldarone CA (2007): Surgical outcomes in the treatment of patients with tetralogy of Fallot and absent pulmonary valve. Eur J Cardiothorac Surg 31(3):354-9

Babu-Narayan SV, Gatzoulis MA: Tetralogy of Fallot. In: Diagnosis and Management of Adult Congenital Heart Disease; hrsg. v. Gatzoulis MA, Webb GD, Daubeney PEF. 2. Auflage; Elsevier Saunders, Philadelphia 2011,316-327

Bailliard F, Anderson R (2009): Tetralogy of Fallot. Orphanet J Rare Dis Jan 13; 4:2

Baron MG (1999): Plain film diagnosis of common anomalies in the adult. Radiol Clin North Am $\underline{37}(2): 401-420$

Blalock A, Taussig HB (1984): The surgical treatment of malformations of the heart in which there is pulmonary stenosis or pulmonary atresia. By Alfred Blalock and Helen B. Taussig. JAMA 251(16):2123-38

Brock RC (1950): Surgery in stenosis of the pulmonary artery. Dia Med 22 (42):166375

Cho JM, Puga FJ, Danielson GK, Dearani JA, Mair DD, Hagler DJ, Julsrud PR, Ilstrup DM (2002): Early and long-term results of the surgical treatment of tetralogy of Fallot with pulmonary atresia, with or without major aortopulmonary collateral arteries. J Thorac Cadiovasc Surg 124(1):70-81

Cooley DA, Hallman GL (1966): Intrapericardial aortic-right pulmonary arterial anastomosis. Surg Gynecol Obstet 122(5):1084-6. 
Duro RP, Moura C, Leite-Moreira A (2010): Anatomophysiologic basis of tetralogy of Fallot and its clinical implications, Rev Port Cardiol 29(4):591-630

Gelb BD, Towbin JA, McCabe ER, Sujansky E (1991): San Luis Valley recombinant chromosome 8 and tetralogy of Fallot: a review of chromosome 8 anomalies and congenital heart disease. Am J Med Genet 40(4):471-6

Hashemzadeh K, Hashemzadeh S (2010): Early and late results of total correction of tetralogy of Fallot. Acta Med Iran 4ㅇ⑵:117-22

Hirsch JC, Mosca RS, Bove EL (2000): Complete repair of tetralogy of Fallot in the neonate: results in the modern era. Ann Surg 232(4):508-14

Kanter KR, Kogon, BE, Kirshbom PM, Carlock PR (2010): Symptomatic neonatal tetralogy of Fallot: repair or shunt, Ann Thorac Surg 89(3):858-63

Kiran VS, Nath PP, Maheshwari S (2011): Spectrum of paediatric cardiac diseases: a study of 15,066 children undergoing cardiac intervention at a tertiary care centre in India with special emphasis on gender, Cardiol Young 21 (1):19-25

Lillehei CW, Cohen M, Warden HE, Read RC, Aust JB, Dewall RA, Varco RL (1955): Direct vision intracardiac surgical correction of the tetralogy of Fallot, pentalogy of Fallot, and pulmonary atresia defects; report of first ten cases. Ann Surg 142(3):41842

Lindberg HL, Saatvedt. K, Seem E, Hoel T, Birkeland S (2011): Single- center 50 years'experience with surgical management of tetralogy of Fallot. Eur $J$ Cardiothorac Surg $\underline{40}(3): 538-42$ 
Marino B, Digilio MC, Grazioli S, Formigari R, Mingarelli R, Giannotti A, Dallapiccola $B$ (1996): Associated cardiac anomalies in isolated and syndromic patients with tetralogy of Fallot. Am J Cardiol 77(7): 505-8

Nollert GD. Däbritz SH, Schmoeckel M, Vicol C, Reichart B (2003): Risk factors for sudden death after repair of tetralogy of Fallot. Ann Thorac Surg 76 $(6): 1901-5$

Potts WJ, Smith S, Gibson S (1946): Anastomosis of the aorta to a pulmonary artery; certain types in congenital heart disease. J Am Med Assoc 132(11):627-31

Richards EG, Zaveri HP, Wolf VL, Klang SH, Scott DA (2011): Delineation of a less than $200 \mathrm{~kb}$ minimal deleted region for cardiac malformations on chromosome 7p22. Am J Med Genet A 155A(7):1729-34

Rudolph AM: Congenital Diseases of the Heart: Clinical-Physiological Considerations. 2. Auflage; Futura Publishing Company, Inc, Armonk (New York) 2001

Rygg ICH, Bertelsen S, Borgeskov S, Fabricius J, Hansen PF, Hasner E, Lauridsen $P$, Melchior J, Sandoe $E$ (1971): The palliativ surgical treatment of tetralogy of Fallot, Dan Med Bull 18 (Suppl 2):59-80

Saeed S, Hyder SN, Sadiq M (2009): Anatomical variations of pulmonary artery and associated cardiac defects in Tetralogy of Fallot. J Coll Physicians Surg Pak $\underline{19}(4): 211-4$

Schumacher G: Fallot-Tetralogie in: Klinische Kinderkardiologie, Diagnostik und Therapie der angeborenen Herzfehler, Schumacher G, Hess J, Bühlmeyer K; 3. Auflage; Springer Verlag, Berlin 2001, 286-297 
Sellors TH (1948): Surgery of pulmonary stenosis; a case in with the pulmonary valve was successfully divided, Lancet 11(6513):988

Siwik ES, Patel RP, Zahka KG, Goldmuntz E: Tetralogy of Fallot. In: Moss and Adam's Heart Disease in Infants, Children and Adolescents, Including the Fetus and Young Adult ; hrsg. v. Allen HD, Gutgesell HP, Clark EB, Driscoll DJ. 6. Auflage; Lippincott Williams and Wilkins, Philadelphia 2001, 880-902

Sousa UM, Lacour-Gayet F, Komiya T, Serraf A, Bruniaux J, Touchot A, Roux D, Petit J, Planché $C$ (1994): Surgery for tetralogy of Fallot at less than six month age. J. Thorac Cardiovasc Surg 107(5):1291-300

Stanley PH, Chartrand C, Davignon A.; Fouron JC, Guerin R, Favreau-Ethier M, Charest J, Kratz C (1981): Palliativ surgery in tetralogy of Fallot. Can J Surg 24(5):475-9

Stewart S, Alexson C, Manning J, Oakes D, Eberly SW (1988): Long-term palliation with the classic Blalock-Taussig shunt. J Thorac Cardiovasc Surg 96(1):117-21

Taussig HB (1947): Diagnosis of the Tetralogy of Fallot and Medical Aspects of Surgical Treatment. Bull N Y Acad Med 23(12):705-18

Tláskal T, Emmrich K, Hucin B, Chares M, Fiser B, Samánek M, Hruda J (1985): Results of total repair of tetralogy of Fallot after previous subclaviopulmonary or aortopulmonary anastomosis.Cor vasa 27(5):353-63

Van Arsdell GS, Maharaj GS, Tom J, Rao VK, Coles JG, Freedom RM, Williams WG, McCrindle BW (2000): What is the optimal age for repair of tetralogy of Fallot. Circulation 102(19 Supp/3): III123-9 
Van Praagh $R$ (2009): The first Stella van Praagh memorial lecture: the history and anatomy of tetralogy of Fallot, Semin Thorac Cardiovasc Surg Pediatr Card Surg Annu 19-38

Vogt J, Wess/hoeft H, Luig H, Schmitz L, De Vivie ER, Weber H, Beuren AJ (1984): The preoperative and postoperative findings in 627 patients with teralogy of Fallot. Thorac Cardiovasc Surg $\underline{32}(4): 234-43$

Waterston DJ (1962): Treatment of Fallot's tetralogy in children under one year of age. Rozhl Chir 41:181-3

Webb GD, Smallhorn JF, Therrien J, Redington A: Congenital Heart Disease. In: Braunwald's Heart Disease: a textbook of cardiovascular medicine; hrsg. v. Libby $P$, Bonow RO, Mann DL, Zipes DP. 8. Auflage; Elsevier Sauders, Philadelphia 2008, 1561-1624

Williams JA, Bansal AK, Kim BJ, Nwakanma LU, Patel ND, Seth AK, Alejo DE, Gott VL, Vricella LA, Baumgartner WA, Cameron DE (2007): Two thousand BlalockTaussig shunts: A six- decade experience. Ann Thorac Surg 84(6):2070-5

Yuan SM, Shinfeld A, Raanani E (2009): The Blalock- Taussig shunt. J Card Surg 24(2):101-8 


\section{Danksagung}

Herrn Priv.-Doz. Dr. med. Theodorus Tirilomis gilt mein besonderer Dank für die Überlassung des Dissertationsthemas sowie die gute wissenschaftliche Betreuung und Unterstützung. 


\section{Lebenslauf}

Am 24. Juni 1980 wurde ich als drittes Kind des Dipl.-Physikers Dr. Theodor Rottwinkel und der Realschullehrerin Annelore Rottwinkel, geb. Bruins, in Göttingen/ Niedersachsen geboren.

Von 1986-1990 besuchte ich die Grundschule und anschließend von 1990-1992 die Orientierungsstufe Adelebsen. Am Otto-Hahn-Gymnasium in Göttingen, das ich von 1992-1999 besuchte, legte ich mein Abitur ab.

Im Sommersemester 2000 nahm ich das Studium der Humanmedizin an der Universität Göttingen auf und erhielt am 29. Mai 2007 meine Approbation als Ärztin.

Am 17. September 2007 begann ich, als Assistenzärztin in der Abteilung Kardiologie am Herzzentrum der Universität Leipzig unter Prof. Dr. Schuler zu arbeiten.

Am 01. Mai 2008 wechselte ich als Assistenzärztin in die Abteilung der Herzchirurgie im Herzzentrum der Universität Leipzig unter Prof. Dr. Mohr.

Ab dem 01.April 2009 wechselte ich in die Orthopädie des Immanuelkrankenhauses Berlin Wannsee unter der Leitung von Prof. Dr. Sparmann. Ab dem 01. Januar 2010 wurde diese Abteilung von Dr. Bauwens geleitet. Am 01. Januar 2012 wechselte ich im gleichen Haus in die Abteilung der oberen Extremität unter der Leitung von Dr. Lautenbach.

Seit dem 09. September 2011 bin ich mit dem Arzt Dr. med. Marek Nowak verheiratet.

Am 29. Juni 2012 ging ich in den Mutterschutz und gebar meine Tochter Karina am 02. August 2012 in Berlin. Die Elternzeit ging bis zum 03. Oktober 2013.

$\mathrm{Ab}$ dem 04. Oktober arbeitete ich in der Abteilung obere und untere Extremität des Immanuelkrankenhauses Berlin Wannsee unter der Leitung von Dr. Naatz, Dr. Berndsen und Garanin.

Seit dem 01. April 2014 bin ich in der Abteilung Hand- und Fußchirurgie des Krankenhauses Waldfriede unter der Leitung von Dr. Lautenbach in Berlin tätig. 